\title{
Stress orientation dependence for the propagation of stacking faults and superlattice stacking faults in nickel-based superalloys
}

\author{
F. D. León-Cázares ${ }^{\mathrm{a}, *}$, F. Monni ${ }^{\mathrm{a}}$, C. M. F. Rae ${ }^{\mathrm{a}}$ \\ ${ }^{a}$ Department of Materials Science and Metallurgy, University of Cambridge, 27 Charles Babbage Rd, \\ Cambridge CB3 OFS, UK
}

\begin{abstract}
Superlattice stacking fault propagation dominates the creep deformation behaviour of nickel-based superalloys at intermediate temperatures. These planar defects may appear under many different configurations depending on the dislocation arrangements and their interactions with the precipitates. Whilst these have been spotted and described before, no systematic way to explain their configurations has been provided. The current study quantifies the types of faults in multiple grains within a tensile crept polycrystalline alloy via a combination of scanning transmission electron microscopy and electron backscatter diffraction. A new defect consisting of a superlattice intrinsic stacking fault in the precipitates and an extrinsic stacking fault in the matrix is observed and a mechanism for its formation is proposed. In combination with data from the literature on single crystals, the results are incorporated into a robust framework to discern the orientation dependencies of these faults. A comprehensive analytical model based on a series of onedimensional force balances on different dislocation configurations is developed first for the case of athermal stacking fault propagation for the cases of cuboidal and spherical precipitates. The model is then extended to include six configurations of superlattice faults and microtwinning. This results in novel mechanistic maps that account for stress, orientation and microstructure, with excellent qualitative agreement with experiments.
\end{abstract}

Keywords: Ni-based superalloys; Creep; Stacking faults; Non-Schmid; Orientation

\section{Introduction}

Stacking faults are planar defects that disrupt the periodic arrangement of crystallographic planes. These have multiple effects on the phase transformations and deformation behaviour of alloys; e.g. in twinning reactions, acting both as obstacles for dislocation glide 1 and accommodating deformation upon propagation 2. Multiple configurations of stacking faults appear in fcc materials (with an ...ABCABC... stacking sequence), but even more develop in nickel-based superalloys due to the presence of short-range order

*Corresponding author. Email: fdl22@cam.ac.uk (C)2020. This manuscript version is made available under the CC-BY-NC-ND 4.0 
within the $\mathrm{L}_{2}$ precipitates. The formulation of a comprehensive theoretical framework to explain the origin of all these configurations as a function of fault energies, microstructure and loading orientation and magnitude remains a challenging task.

Slip in the fcc $\gamma$ matrix occurs mainly on the 12 independent $\frac{a}{2}\langle 110\rangle\{111\}$ slip systems 3. According to Frank's rule, each of these perfect dislocations may split into two closely spaced $\frac{a}{6}\langle 112\rangle$ Shockley partials bounding an intrinsic stacking fault (ISF) [4] $(\ldots \mathrm{ABC}\lrcorner \mathrm{AC}\llcorner\mathrm{ABC} \ldots)$. The dissociation width between these is typically high due to the low stacking fault energy of these heavily alloyed materials. Under some loading conditions a stacking fault may extend indefinitely if the leading partial continues to glide while the trailing one is pinned [5]. This defect becomes an extrinsic stacking fault (ESF) (...ABC $\lrcorner \mathrm{ACBC}\llcorner\mathrm{ABC} \ldots)$ if an identical partial glides on an adjacent $\{111\}$ plane, and a twin (...ABC $\lrcorner \mathrm{BACBAC}\llcorner\mathrm{ABC} \ldots)$ if the process is repeated on additional layers [6.

Within $\gamma^{\prime}$ precipitates, glide of a perfect dislocation leaves behind an antiphase boundary (APB) which is then reverted back to the original structure by a trailing dislocation. Alternatively, deformation in $\gamma^{\prime}$ may be accommodated via the formation and propagation of superlattice stacking faults, i.e. superlattice intrinsic stacking fault (SISF) and superlattice extrinsic stacking fault (SESF), driven by glide of combinations of $\frac{a}{6}\langle 112\rangle$ partial dislocations. These depend upon two diffusion-controlled mechanisms. Firstly, the segregation of $\gamma$-forming elements ahead of the stacking faults [7] lowers the energy of the defects 8 , which also lowers the shear resistance for the leading dislocations. Secondly, local atomic reordering transforms the initially formed high energy defect into a superlattice fault of much lower energy [9, 10. These thermally activated mechanisms dominate the deformation behaviour during creep at intermediate temperatures and low strain rates [11, where dislocation climb is not predominant. Observations of elemental segregation to stacking faults have, so far, been confined to the $\mathrm{L}_{2}$ phase [12.

A number of mechanisms have been proposed for the creation of the superlattice faults. Superpartials with a $\frac{a}{3}\langle 112\rangle$ Burgers vector were thought to drive SISF propagation, although their existence on a single plane would produce a wrong stacking on the atomic layers [9. Vorontsov et al. [13] were first to prove via high resolution transmission electron microscopy (TEM) that the leading ends of the SISFs and SESFs have a different character to that of the extended faults and are bounded by closely spaced partials gliding on adjacent planes. This suggests the concomitant glide of dissimilar Shockley partials is a possible pathway for the formation of these defects.

The spatial configuration of the superlattice faults also varies considerably. They may appear either isolated within the precipitates or as extended bands running through both phases [13, 14. A more complex dislocation arrangement is that of a stacking fault ribbon (SF ribbon), where the SISF-APB-SESF alternating character results from the cooperative motion of partial dislocations [15, 16]. Another deformation mechanism driven by partial dislocations is the formation and storage of microtwins [9, 17, with a typical thickness of under 50 atomic planes [10, also formed by glide of identical Shockley partials on adjacent planes [9. An initial pseudo-twin (with an orthorhombic structure) is then transformed back to the original $\mathrm{L}_{2}$ structure by a local atomic reordering process. Segregation of $\gamma$-forming elements has also been observed ahead of the leading partials driving microtwin propagation and thickening. As these defects grow in size, segregation remains present at the twin boundaries but not within the twinned regions [18.

Investigations on the stress orientation dependence for the formation and propagation 
of ISFs have been performed mainly via simulations of individual dislocations by Douin et al. [19] and subsequent studies [5, 20, 21]. However, rationalising the effects of loading orientation on the propagation of superlattice faults is considerably more difficult due to the different Shockley partials involved.

A recently developed orientation analysis framework 22] introduced a way of studying slip via the angle $\phi$ between the resolved shear stress on an octahedral plane and its closest $\frac{a}{2}\langle 110\rangle$ slip direction. This angle ranges between $-30^{\circ}$ and $30^{\circ}$, pointing in the direction of the Burgers vector of a leading partial or a trailing partial, respectively. This geometry is schematically shown in Figure 1(a). Under uniaxial tension, $\phi=-30^{\circ}$ for loading along an orientation on the line from $\langle 011\rangle$ to $\langle\overline{1} 11\rangle$ and $\phi=30^{\circ}$ on the $\langle 001\rangle$ direction; these reverse upon uniaxial compression. The reader is referred to the original work [22] for a more detailed description. This framework will be particularly useful in this context as it simultaneously captures the stress states of all the slip systems on a given slip plane, including the Schmid stress pushing the dislocations and the Escaig stress (that perpendicular to the Schmid stress and on the same slip plane) influencing the stacking fault width.

Figure 1(b) includes the results of multiple studies on uniaxial creep in single crystal nickel-based superalloys, both in tension and compression, according to the most frequent mechanism observed in each condition as a function of angle $\phi$. The results were included when there was a quantitative analysis or when it was clearly stated that a specific mechanism appeared more than others. This description unequivocally shows the orientation dependence and the proximities between the mechanisms of SISF and SF ribbon, as well as SESF and microtwinning. Note that most of the studies have been performed in orientations close to $\langle 001\rangle,\langle 011\rangle$ and $\langle 111\rangle$; thus, there is insufficient data for loading around $\phi=0$, orientations located roughly between the $\langle 012\rangle$ and $\langle 123\rangle$ directions.

The current work expands on this analysis by considering the effects of stress orientation of multiple grains within a polycrystalline superalloy. A series of one-dimensional equations are then developed to set the basis of an analytical model that explains the appearance of multiple stacking fault configurations as a function of stress, loading orientation and microstructure.

\section{Material and methods}

The material investigated in this study was a development composition 30 produced via powder metallurgy. Material from compacted and extruded -270 mesh $(53 \mu \mathrm{m})$ powder was isothermally forged and solution heat treated. It subsequently received a post-solution heat treatment of 2 hours at $850{ }^{\circ} \mathrm{C}+4$ hours at $800{ }^{\circ} \mathrm{C}$. An interrupted tensile creep test was performed at $700{ }^{\circ} \mathrm{C}$ and $800 \mathrm{MPa}$ at Swansea Materials Research \& Testing Ltd (SMaRT). A $0.23 \%$ total strain was selected anticipating the formation of enough stacking faults for the analysis while also minimising lattice rotation.

TEM samples were cut from the gauge length of crept samples into $3 \mathrm{~mm}$ discs perpendicular to the loading axis. The discs were ground to a P2500 grit and electropolished with a Tenupol -5 at $-5{ }^{\circ} \mathrm{C} \pm 1{ }^{\circ} \mathrm{C}$ in a $6 \%$ perchloric acid in methanol solution. TEM characterisation was performed with a FEI Tecnai Osiris 80-200 operating at $200 \mathrm{kV}$ in a single sample. Multiple stacking faults in grains close to the hole were imaged through scanning TEM (STEM) in two beam conditions with a high angle annular dark field 
(a)

(b)
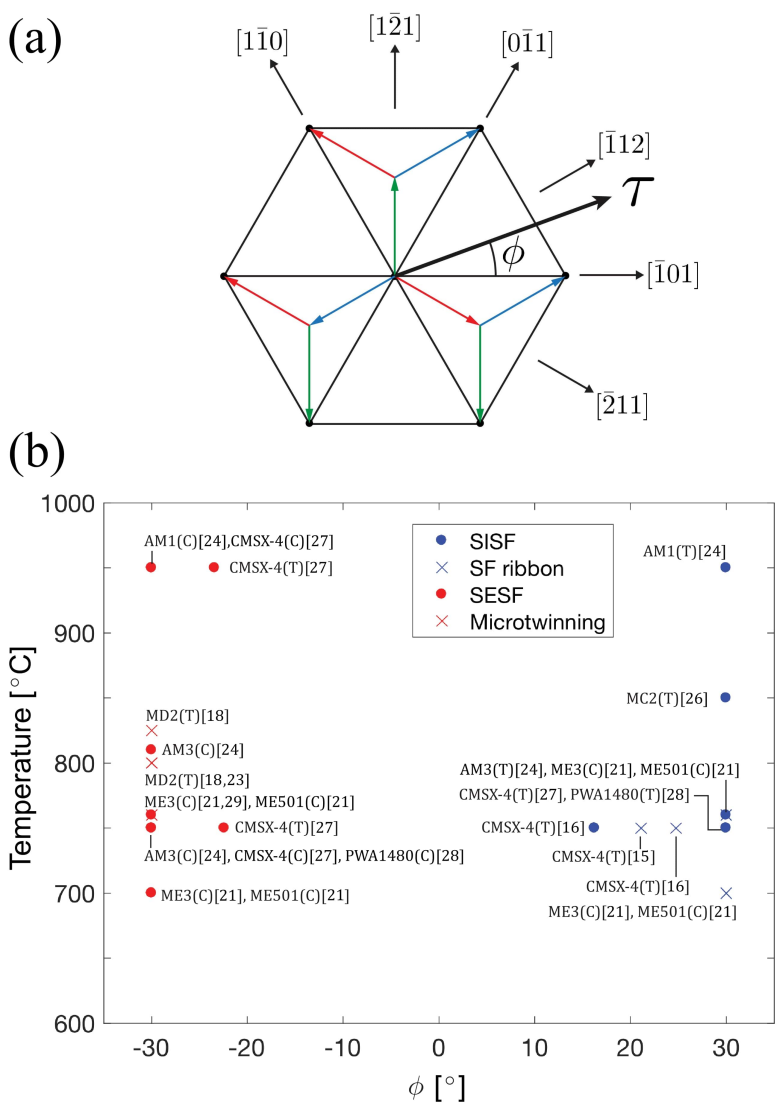

Figure 1: (a) Schematic diagram of a $\{111\}$ plane, where the atoms sit on the corners of the triangles 22 . The arrows show the routes for an atom in the middle to move to a neighbouring position. Angle $\phi$ is that between the shear stress and its closest $\langle 1 \overline{1} 0\rangle$ direction. (b) Map of the most frequently observed stacking fault shearing mechanism as a function of angle $\phi$ and temperature in a series of uniaxial creep studies in single crystal alloys [15, 16, 18, 21, 23, 29]. Each annotation includes the alloy, loading mode and reference.

(HAADF) detector. The character of the stacking faults was determined following the methodology by Williams and Carter [31: placing the origin of the g-vector at the centre of the fault in a dark field micrograph, it points towards the bright outer fringe if it is intrinsic or away from it if it is extrinsic for 200, 222 and 440 reflections; a reverse correlation occurs for 111, 220 and 400 reflections. Stacking faults near grain boundaries were avoided as deviations from the stress in the interior of the grains are pronounced in these regions 32 .

Scanning electron microscopy (SEM) was performed in the same sample with a ZEISS GeminiSEM 300 operating at $25 \mathrm{kV}$. The backscattered electron detector (BSD) was used to image the microstructure and the average size and volume fraction of the secondary $\gamma^{\prime}$ precipitates were estimated via a particle analysis in the ImageJ software package. Electron backscatter diffraction (EBSD) data was acquired with an Oxford Instruments 
Symmetry EBSD detector. Orientation data was collected using Oxford Instruments AZtechHKL software and subsequently analysed using MATLAB with the MTex v5.1.1 package 33 . The grain orientations were calculated using a $1^{\circ}$ misorientation threshold and data was de-noised using a spline smoothing function in order to minimise the effect of unindexed pixels and wild spikes [34].

\section{Results}

The strain curve up to the point where the test was interrupted behaves linearly with a strain rate of $\sim 2.7 * 10^{-8} \mathrm{~s}^{-1}$. Characterisation of the material reveals an average grain size of 30-40 $\mu \mathrm{m}$. The microstructure, shown in Figure 2(a), consists of flowery secondary $\gamma^{\prime}$ with an average size of $0.25 \mu \mathrm{m}$ and volume fraction of $44.7 \%$, and rounded tertiary precipitates in-between. The majority of grains contain stacking faults and dislocations in varying quantities. An example of this can be seen in Figure 2(b), although most grains have fewer defects. Figure 2(c) shows the EBSD scan of the TEM sample with grains coloured according to their crystallographic direction parallel to the loading axis. Other regions of the sample were too thick to be imaged in the TEM. The grains numbered are those where the stacking faults were quantified, which cover most of the stereographic triangle in Figure 2(d).

The number and type of stacking faults observed are summarised in Table 1 together with the value of the angle $\phi$ on their slip plane. Individual slip plane orientations are quantified in grains with stacking faults on two non-coplanar slip systems. All configurations recorded correspond to the secondary precipitates. Note that all tertiaries observed showed either extended faults (continuous through the matrix and precipitates) or no faults at all. Examples of the faults encountered are shown in Figure 3(a-g). Three instances of a new fault configuration were found in grain 9, consisting of SISFs in the precipitates with an ESF in the matrix (Figure $3(\mathrm{~g})$ ). To the knowledge of the authors this type of fault has not been reported in the literature before.

Table 1: Number and type of superlattice stacking faults quantified in each grain.

\begin{tabular}{|c|c|c|c|c|c|c|c|c|}
\hline Grain & $\phi$ & Isolated SESF & $\mathrm{SESF}+\mathrm{ESF}$ & $\mathrm{SESF}+\mathrm{ISF}$ & Isolated SISF & $\mathrm{SISF}+\mathrm{ISF}$ & SF ribbon & $\mathrm{SISF}+\mathrm{ESF}$ \\
\hline \multirow[t]{2}{*}{1} & $-30^{\circ}$ & - & - & - & 6 & 1 & - & - \\
\hline & $-25.9^{\circ}$ & - & 1 & - & 1 & - & - & - \\
\hline 2 & $-25.6^{\circ}$ & 3 & 1 & - & 3 & - & 1 & - \\
\hline \multirow[t]{2}{*}{3} & $-18.2^{\circ}$ & - & - & 1 & 3 & - & - & - \\
\hline & $-17.1^{\circ}$ & 2 & - & - & - & - & - & - \\
\hline 4 & $-15.1^{\circ}$ & 5 & 2 & 1 & 10 & 4 & - & - \\
\hline 5 & $-12.3^{\circ}$ & 1 & - & - & 4 & 2 & 2 & - \\
\hline \multirow[t]{2}{*}{6} & $-11.1^{\circ}$ & - & 2 & 1 & 2 & 1 & - & - \\
\hline & $-10.5^{\circ}$ & 3 & - & 1 & 1 & - & - & - \\
\hline 7 & $-6.5^{\circ}$ & - & 7 & - & 10 & 1 & 4 & - \\
\hline 8 & $-1.8^{\circ}$ & 4 & 4 & - & 1 & 4 & - & - \\
\hline 9 & $0.9^{\circ}$ & 3 & 1 & - & - & 3 & - & 3 \\
\hline 10 & $4.1^{\circ}$ & - & - & - & - & - & - & - \\
\hline 11 & $6.6^{\circ}$ & - & - & - & 1 & 5 & - & - \\
\hline 12 & $26^{\circ}$ & - & - & - & 3 & 1 & 1 & - \\
\hline \multirow[t]{2}{*}{13} & $29.9^{\circ}$ & 3 & - & - & 1 & - & 2 & - \\
\hline & $16.4^{\circ}$ & 1 & - & - & 2 & - & 1 & - \\
\hline
\end{tabular}

In many cases a single fault is observed to change its configuration, e.g. from an isolated SESF to an extended SESF + ESF. In these situations the most predominant 

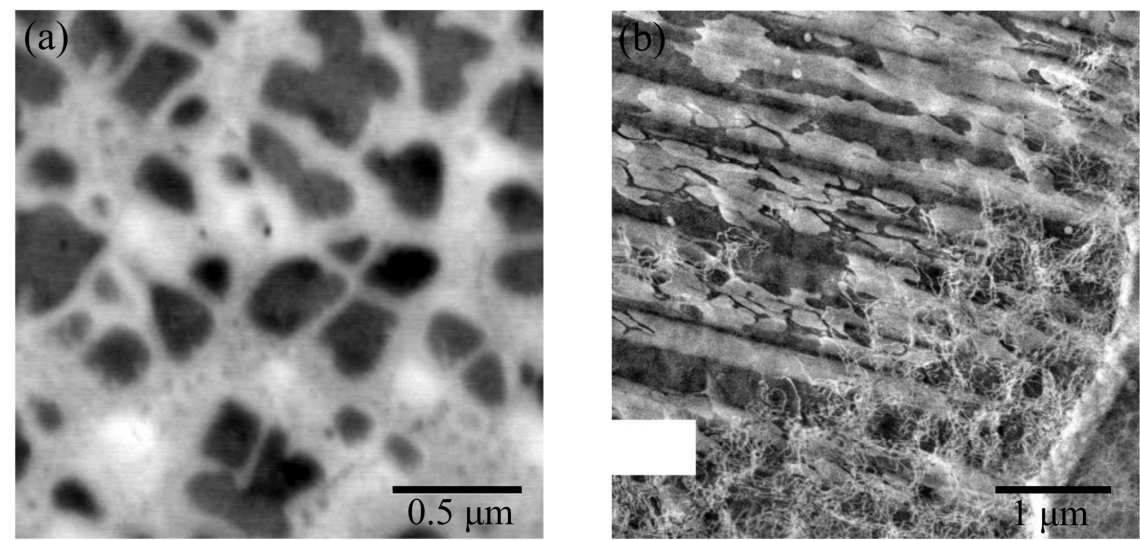

(c)

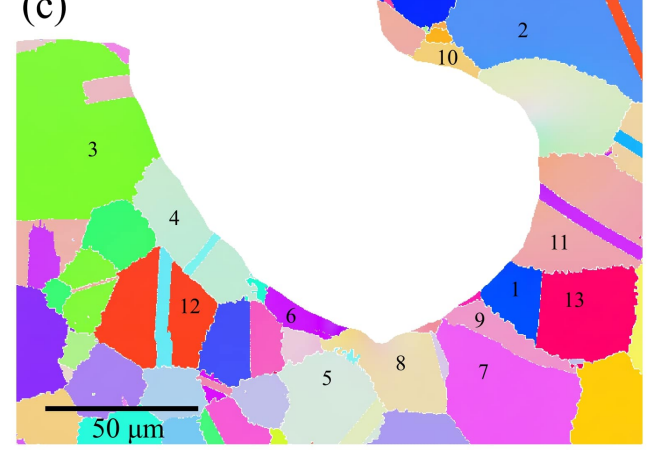

(d)

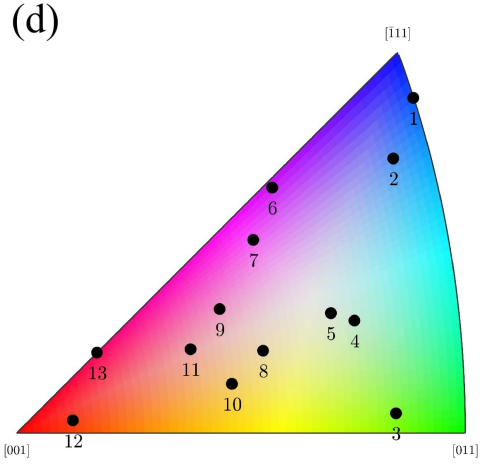

Figure 2: (a) BSD micrograph of the microstructure showing the precipitate morphology, (b) STEM micrograph of a grain showing dislocations near the grain boundary and stacking faults propagating towards the interior, and (c) EBSD map of the deformed TEM sample colour-coded according to the grain orientations in (d) an inverse pole figure related to their crystallographic direction parallel to the loading axis. Numbered grains are those were the stacking faults were quantified.

configuration is the one recorded in Table 1. SF ribbons were only considered as such when both the SISF and SESF segments were seen in the micrograph. Note that these defects are vulnerable to false negative errors if only the SISFs or SESFs segments of the fault appear in the sample.

Figure 4 maps the value of $\phi$ onto the stereographic triangle (except for grain 13 in which another slip plane contains the highest shear stress) together with pie charts with the quantified fractions of faults per grain. Most grains contain SISFs, whereas SESFs appear more often for lower $\phi$ angles. The trend is similar to that in the single crystal data, although with more scatter in the types of faults. A notable exception is grain 1, where intrinsic faults appear in higher amounts than the expected extrinsic ones.

Perfect $\frac{a}{2}\langle 110\rangle$ dislocations constantly appear near the grain boundaries but only populate the matrix in the interior of the grains in a few instances. The highest dislocation densities developed in grains 1, 2, 3, 10 and 11; the last two have the highest Schmid factors for perfect dislocations. Only in grain 10 no stacking faults are observed, with the exception of two short SESFs extending less than a micron away from grain boundaries. 

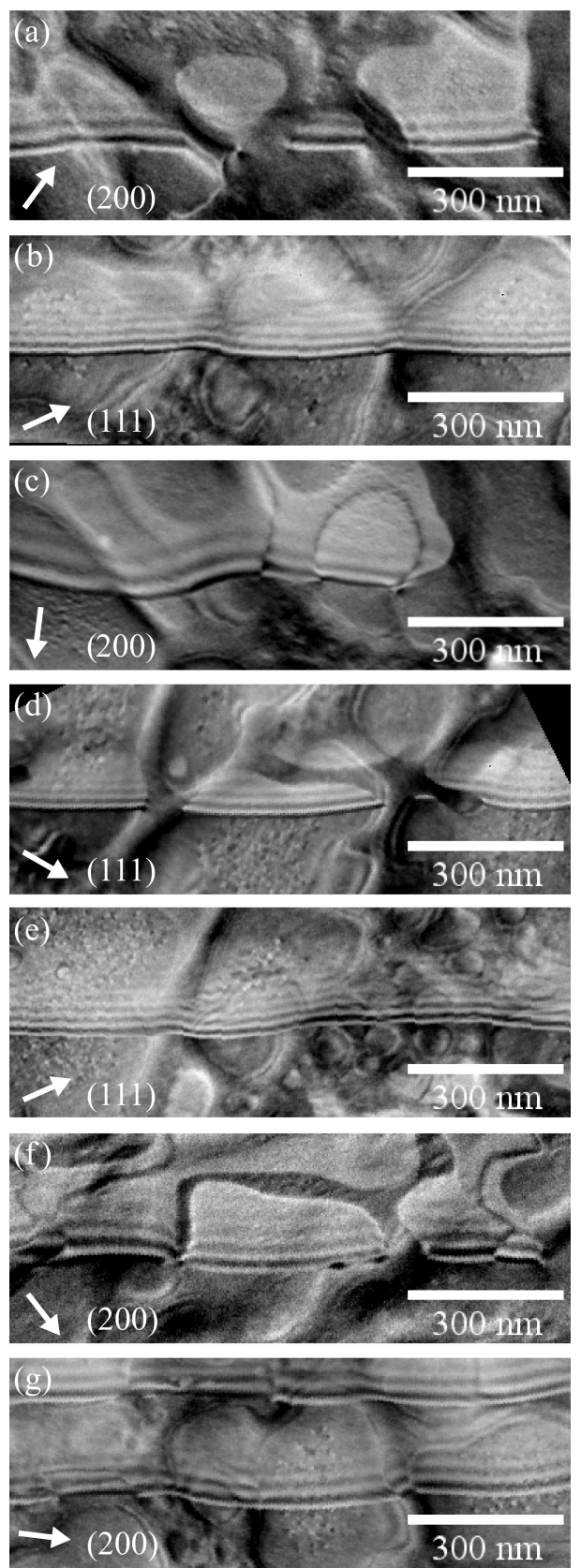

Figure 3: STEM micrographs of an (a) isolated SESF, (b) extended SESF + ESF, (c) extended SESF + ISF, (d) isolated SISF, (e) extended SISF + ISF, (f) SF ribbon and an (g) extended SISF + ESF. The corresponding reflections are drawn in each micrograph.

Thus, no planar faults are recorded for this grain. 


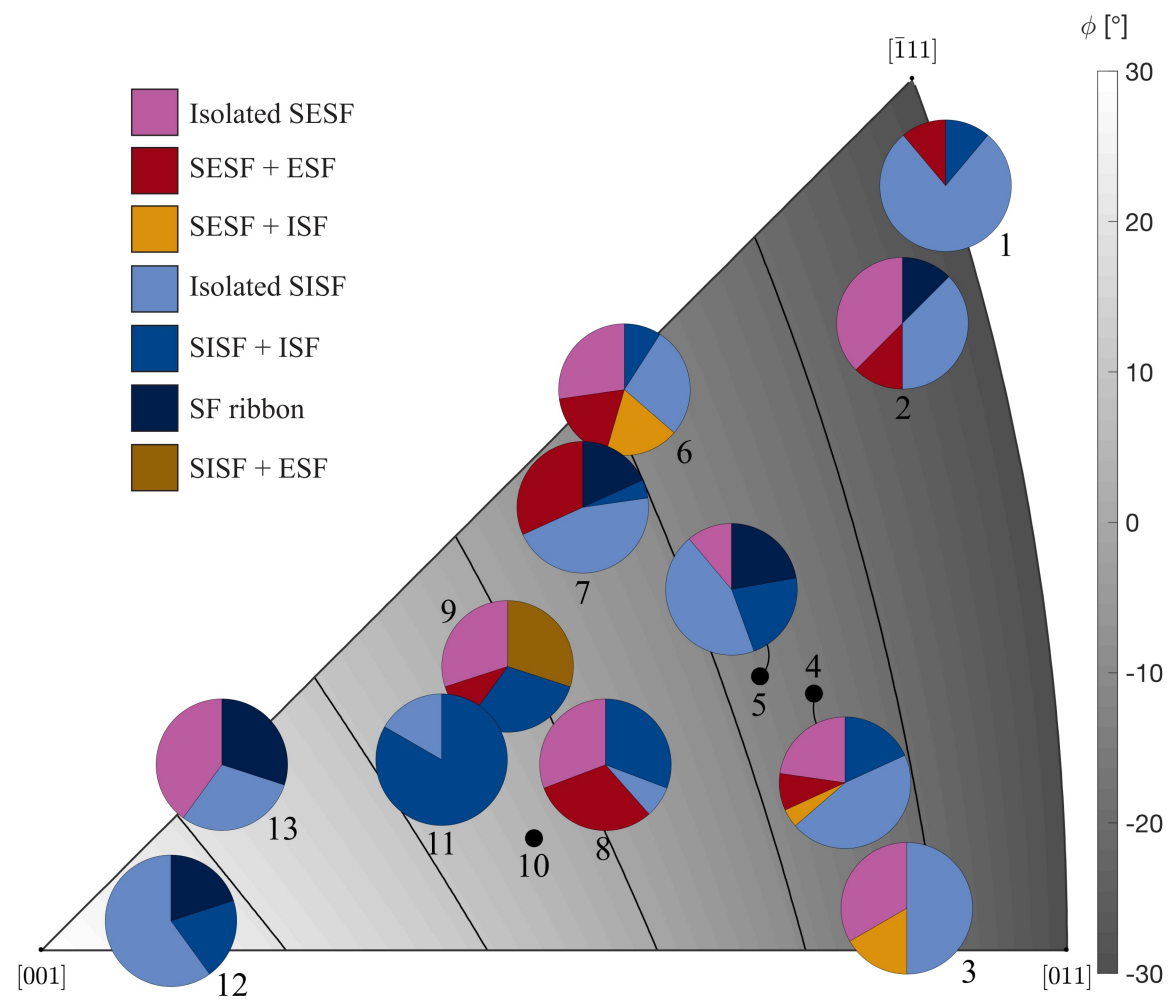

Figure 4: Stereographic triangle with the value of $\phi$ and pie charts with the fractions of each superlattice fault configuration observed in each grain. No defects are registered for grain 10.

\section{Analytical model}

The following analysis is based on the framework presented in reference [22]. Consider the coordinate system in Figure 5 positioned with respect to a $\frac{a}{2}\langle 110\rangle\{111\}$ dislocation so that the $x$-axis is aligned with its Burgers vector $\bar{b}$ and the $z$-axis is perpendicular to its slip plane. Regardless of the dislocation character $\theta$, the dislocation moves in the $\hat{y}$ direction and the Burgers vectors of the corresponding leading and trailing Shockley partials point at $-30^{\circ}$ and $30^{\circ}$ angles from $\bar{b}$, respectively.

Consider now an infinitely long dissociated dislocation in a frictionless and otherwise perfect fcc crystal. The forces per unit length experienced by the partials are $F_{1}=$ $F_{a 1}-\gamma_{I S F}+F_{\text {int }}$ and $F_{2}=F_{a 2}+\gamma_{I S F}-F_{\text {int }}$, where the subindices ${ }_{1}$ and 2 refer to the leading and trailing partials, $F_{a}$ are the forces from the applied stress, $\gamma_{I S F}$ the intrinsic stacking fault energy, $F_{\text {int }}=A / d$ the dislocation interaction force with

$$
A=\frac{2-\nu-2 \nu \cos 2 \theta}{8 \pi(1-\nu)} G b_{p}^{2}
$$

$d$ is the distance between the partials, $G$ the shear modulus, $b_{p}=b / \sqrt{3}$ the Burgers vector of a partial dislocation and $\nu$ the Poisson's ratio. In the current coordinate system, 


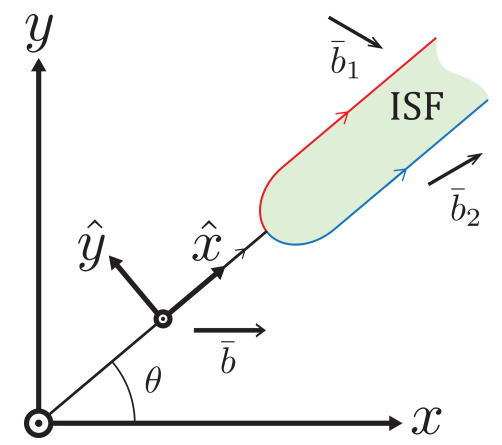

Figure 5: Coordinate systems for a perfect dislocation and its Shockley partials [22]. The arrows along the dislocations represent the line vectors.

the Peach-Koehler forces from the applied stress are $F_{a 1}=\frac{b}{2} \tau_{S}-\frac{b}{2 \sqrt{3}} \tau_{E}$ and $F_{a 2}=$ $\frac{b}{2} \tau_{S}+\frac{b}{2 \sqrt{3}} \tau_{E}$, where the Schmid stress $\tau_{S}$ drives perfect dislocation glide and the Escaig stress $\tau_{E}$ pushes both partials in opposite directions. A force balance $F_{1}=F_{2}$ gives the stacking fault width [22, 35]

$$
d_{E}=\frac{A}{\gamma_{I S F}+\frac{b}{2 \sqrt{3}} \tau_{E}} .
$$

However, both partials will continue to move in the same direction and at the same speed, unless $\tau_{S}=0$. In order for the stacking fault to reach a true stable and immobile width, i.e. $F_{1}=F_{2}=0$, at least one more force in the form of an obstacle is required.

Forces opposing dislocation glide can appear in two forms. Firstly, as friction that acts along every segment of a dislocation. The friction forces per unit length on the partials $F_{f 1}$ and $F_{f 2}$ are different from that on a perfect dislocation $F_{f}$ as they have different dislocation characters [36. Secondly, forces $R_{1}$ and $R_{2}$ from an obstacle or series of obstacles (precipitates in the case of nickel-based superalloy) inhibit dislocation motion either when they come into contact with the dislocation or, more realistically, by introducing a stress gradient into the crystal.

Consider now the dissociated infinitely long dislocation but this time in a crystal with friction and with the leading partial pushing against an obstacle, so that a new force balance arises

$$
\begin{gathered}
F_{a 1}+F_{i n t}-\gamma_{I S F}-F_{f 1}-R=0 \\
F_{a 2}-F_{i n t}+\gamma_{I S F} \pm F_{f 2}=0,
\end{gathered}
$$

where $R$ is the reaction force exerted by the obstacle and the \pm sign accounts for the two possible directions in which the trailing partial could move. Solving this system of equations results in $R=b \tau_{S}-F_{f 1} \pm F_{f 2}$ and

$$
d_{\sigma}=\frac{A}{\gamma_{I S F}+\frac{b}{2} \tau_{S}+\frac{b}{2 \sqrt{3}} \tau_{E} \pm F_{f 2}}
$$

as the new range for a stable stacking fault width. This result shows how the Schmid stress can have an effect on $d$ when considering the presence of obstacles, but unlike the Escaig stress, $\tau_{S}$ will only decrease its value. 


\subsection{Athermal stacking fault propagation}

Athermal propagation is used here to refer to the glide of partial dislocations driven solely by the stress field without any effect of atomic diffusion. Analogous to classical theory of precipitate hardening, it is possible to devise multiple paths individual Shockley partials may take to overcome an obstacle. In nickel-based superalloys with a large $\gamma \prime$ volume fraction cuboidal precipitates are typically looped around via glide through the narrow $\gamma$ channels in-between. Alternatively, alloys with a lower volume fraction have smaller and more spherical precipitates that can be surpassed via shearing or Orowan looping. Depending on the sequence and whether the trailing partial is blocked or not, the pathways drawn in Figure 6 can take place, leaving behind different defects. The nomenclature used here for the different mechanisms follows this sequence: a number indicating which partial it refers to, with following letters referencing the sequence of mechanisms used (s for shearing and $\ell$ for looping); e.g. (2sl) refers to looping of the trailing partial around a previously sheared precipitate. Appendix A describes in more detail the critical forces required to overcome these obstacles.

Consider the conditions required for the athermal propagation of a stacking fault subject to obstacles and friction forces as described above. The most favourable scenario for the decorrelation of the partials is that with a stress state that promotes the formation of an infinitely long stacking fault. This will occur when two conditions are met: $(\mathrm{C} 1)$ that the leading, and $(\mathrm{C} 2)$ trailing partials, will always have the force to surpass the obstacles in the $-\hat{y}$ and $\hat{y}$ directions, respectively. These conditions must apply even after the partials are completely decorrelated, i.e. when $d \rightarrow \infty$ and $F_{\text {int }}=0$, and can be written as

$$
\mathrm{C} 1: F_{a 1}-\gamma_{I S F}-F_{f 1} \geq R_{1}
$$

and

$$
\mathrm{C} 2: F_{a 2}+\gamma_{I S F}+F_{f 2} \leq-R_{1} .
$$

In this situation the trailing partial behaves as the leading one but glides in the opposite direction. A closer look at these conditions shows that whenever C2 holds true, then C1 does too.

If $\mathrm{C} 2$ is false, then the trailing partial will move in the $\hat{y}$ direction until it encounters obstacles. A semi-infinite stacking fault arises if two conditions are true: $(\mathrm{C} 1)$ that the leading partial will always have the force necessary to overcome the precipitates, and (C3) that the trailing partial will never overcome the obstacles that have been previously surpassed by the leading one, i.e.

$$
\text { C3 }: F_{a 2}+\gamma_{I S F}-F_{f 2}<R_{2} .
$$

If only $\mathrm{C} 1$ is false, then the leading partial will eventually get blocked and the resulting finite stacking fault will be immobile. If only $\mathrm{C} 3$ is false, then the trailing partial will start surpassing the precipitates and the stacking fault will become mobile.

If both conditions $\mathrm{C} 1$ and $\mathrm{C} 3$ are false, then the outcome will depend on which of the two events occurs first. Adding the interaction force with its corresponding sign to both conditions and turning them into equalities leads to the critical widths at which the stacking fault will be blocked

$$
d_{\text {blocked }}=\frac{A}{-F_{a 1}+\gamma_{I S F}+R_{1}+F_{f 1}}
$$




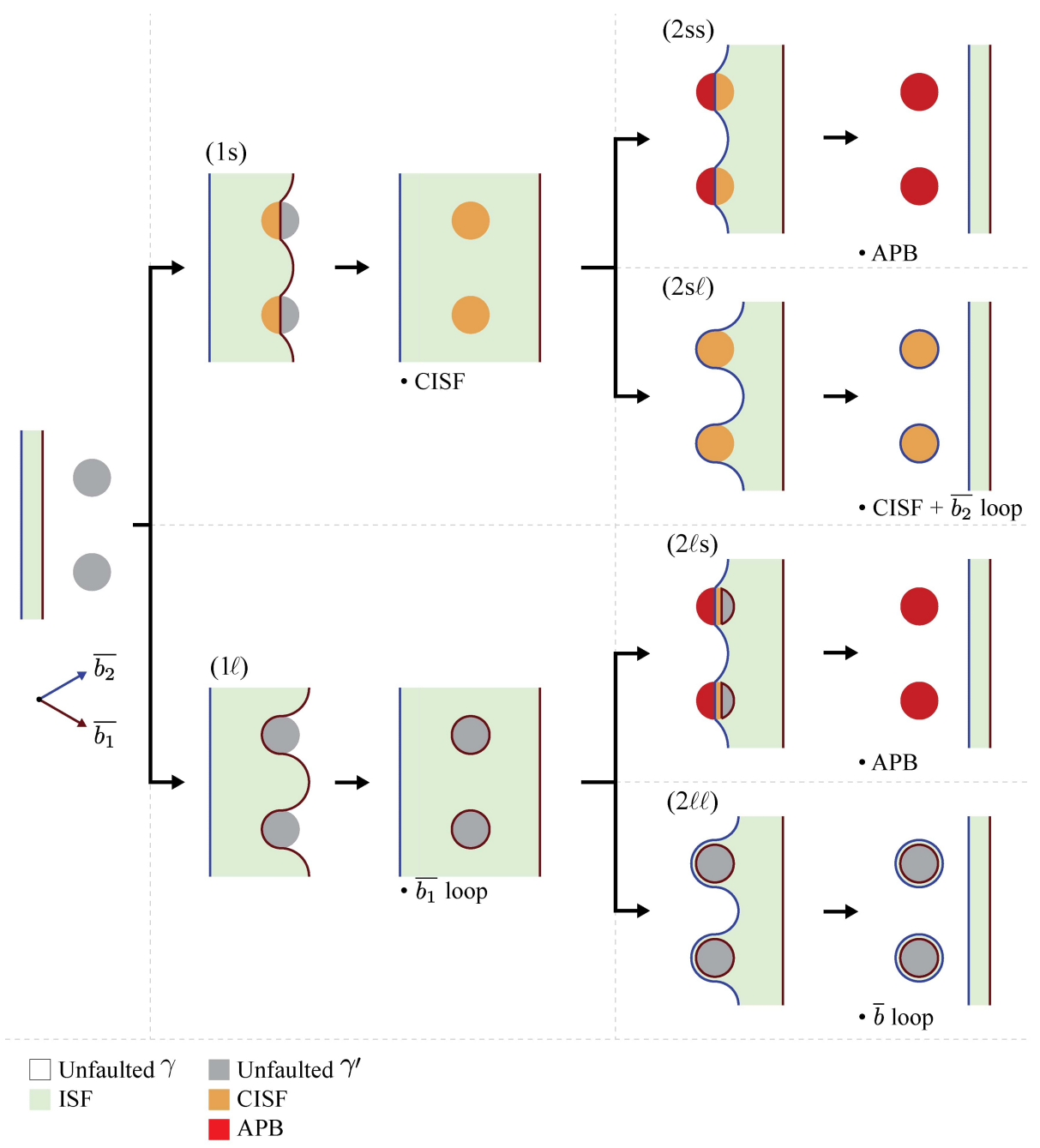

Figure 6: Schematic diagrams of the mechanisms for Shockley partial precipitate bypassing where dislocations glide from left to right. The defects left after the sweeping of each partial are annotated.

or become mobile

$$
d_{\text {mobile }}=\frac{A}{F_{a 2}+\gamma_{I S F}-R_{2}-F_{f 2}} .
$$

Combining them and rearranging the terms results in a new condition

$$
\mathrm{C} 4: \tau_{S}>\frac{1}{b}\left(R_{1}+R_{2}+F_{f 1}+F_{f 2}\right)
$$

for the resulting stacking fault to be mobile.

After the width $d_{\text {mobile }}$ is reached, the force on the leading partial is still bigger than that on the trailing one, thus continuing to extend the stacking fault. This will stop 
whenever the leading partial cannot surpass the obstacles any longer $\left(d=d_{\text {blocked }}\right)$ or when both dislocations feel the same force and start moving in a correlated way. This last condition will occur at a separation of

$$
d_{\text {correlated }}=\frac{A}{\gamma_{I S F}+\frac{b}{2 \sqrt{3}} \tau_{E}+\frac{R_{1}-R_{2}+F_{f 1}-F_{f 2}}{2}} .
$$

Whenever condition $\mathrm{C} 4$ is met, then $d_{\text {correlated }}<d_{\text {blocked }}$, meaning that overall correlated motion of partials will happen with a stacking fault width given by equation (11). As expected, if $R_{1}=R_{2}$ and $F_{f 1}=F_{f 2}$, then the mobile stacking fault width will be $d_{E}$. Moreover, the condition for the uncorrelated motion of partials in the $\hat{y}$ direction becomes

$$
\mathrm{C} 5: \tau_{E} \leq-\frac{\sqrt{3}}{b}\left(2 \gamma_{I S F}+R_{1}-R_{2}+F_{f 1}-F_{f 2}\right) .
$$

With this set of conditions it is possible to estimate the behaviour of the dislocation under any stress state. By rearranging all of them in the form of equations one gets a series of straight lines that subdivide a map of $\tau_{S}$ vs. $\tau_{E}$ into five main dislocation regimes, as shown in Figure 7. Diagrams similar to this one have been obtained before via dislocation dynamics simulations [19, 37, (more details in Section 5). Stress orientation maps like those in reference 22] can then be superimposed onto such a plot to determine the behaviour of each slip system. Note that this is only possible if the obstacles offer the same resistance in every direction, which would not be the case if $R_{1}$ had an orientation dependence, e.g. for non-spherical precipitates with a preferential orientation or for forest dislocations that do not provide the same glide resistance in different slip systems due to latent hardening 38 .

Most of the features in Figure 7 are constrained due to the nature of the conditions. The slopes of the inclined lines are fixed at $\sqrt{3}$ and $-\sqrt{3}$ as they are forces that oppose glide of the partials. The position of point $P$ as determined by equations $(10)$ and $(12)$ is critical to the occurrence of athermal stacking faults as will be discussed later. It must also be noted that condition $\mathrm{C} 4$ is different from the boundary for glide resistance of a perfect dislocation $R$, i.e. $R \neq R_{1}+R_{2}$. This is due to the difference between the tensions of a Shockley partial and a perfect dislocation [39. If the partials are not decorrelated yet, this boundary and the regions around point $P$ should be modified accordingly.

\subsection{Thermally activated stacking fault propagation}

At higher temperatures, other mechanisms of stacking fault propagation are facilitated by atomic diffusion. To the knowledge of the authors no general explanation has yet accounted for all the configurations that have been observed experimentally. In this section we rationalise the effects of orientation and microstructure underlying the propagation of these defects, which include:

- Isolated SISFs

- Extended SISFs + ISF

- Stacking fault ribbons

- Isolated SESFs 


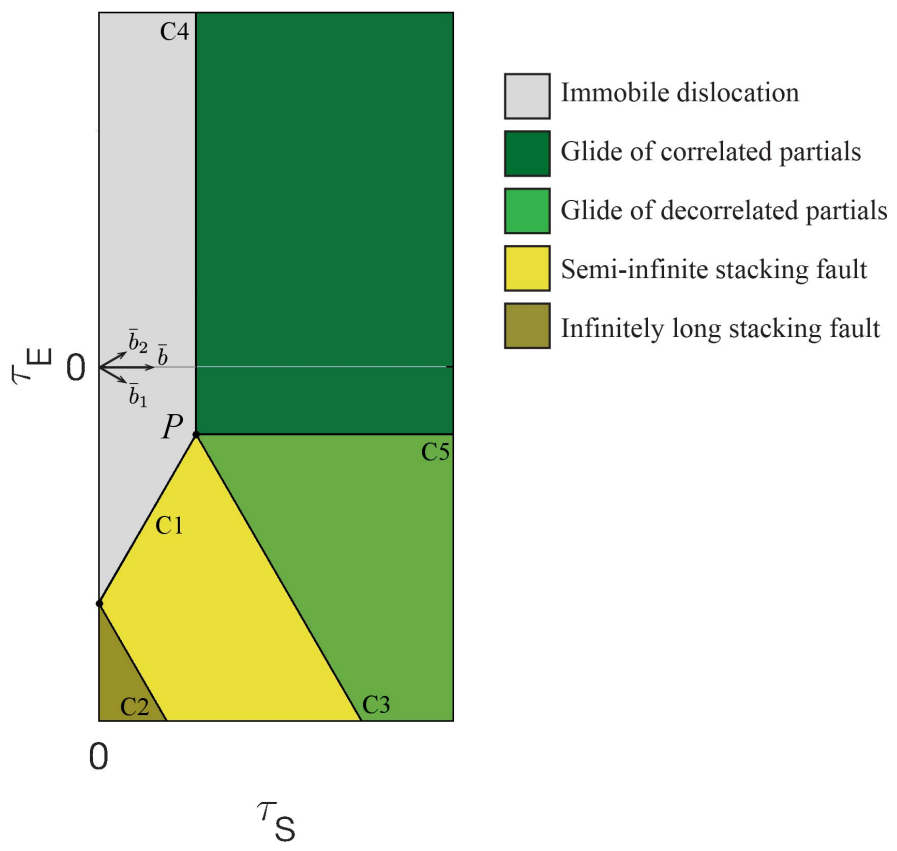

Figure 7: Schematic plot of the different athermal glide regimes for a dissociated $\frac{a}{2}\langle 110\rangle\{111\}$ dislocation with an aspect ratio of 1:1. The boundaries are defined by conditions C1-C5.

- Extended SESFs + ESF

- Extended SESFs + ISF

- Microtwins

The observed SISF + ESF configuration is not included in this analysis because its dislocations have not been identified yet, but comments and a proposed mechanism for its formation and propagation are described in Section 5. Segregation assisted shear by perfect $\frac{a}{2}\langle 110\rangle$ dislocations is also not included as it was not experimentally quantified, but its occurance may have a similar orientation dependence to that of athermal glide of correlated partials (equation (10) ) with a lower stress threshold.

Modelling in this section is restricted to the propagation of faults and it leaves for later the nucleation mechanisms, which are not yet fully understood. The segregation-assisted plasticity theory 23 has shown that $\gamma$-stabiliser elements diffuse towards such faults, lowering their energy penalties 8 . In the current framework this constitutes a difficulty as it is hard to pinpoint what the actual energies of the different faults are. The values from the literature used here may be considered upper bounds as the real faults would form in a more $\gamma$ like structure due to elemental segregation. Also note that the unstable energies from the generalised stacking fault energy should be considered, rather than only the stable points $40-43$. For simplicity and due to the lack of concentration dependent data, all the forces coming from planar defects in this work are assumed to come from 
the stable fault energies. Moreover, the description for large cuboidal precipitates is used in this section for simplicity, disregarding the effect of tertiary precipitates and the additional tension from the segments in the matrix. Athermal bypassing of the precipitates is then only opposed by the stresses required to glide through a $\gamma$ channel, $R_{1 c}$ and $R_{2 c}$, as defined in Appendix A (equations A.1 and A.2 ).

\subsubsection{SISF-related mechanisms}

Consider first the case of a SISF formed by glide of a dislocation of Burgers vector $\frac{a}{3}\langle 112\rangle$, depicted in Figure 8. It is envisioned that two coplanar dislocations with different leading but similar trailing partials will first meet on adjacent planes at a $\gamma / \gamma /$ interface. Glide into the precipitate of a full dislocation and an additional leading partial will result in an arrangement such as that in Figure 8(b), where a complex extrinsic stacking fault (CESF) is followed by an APB. A local diffusive reordering scheme is necessary to transform the latter into a SISF with a much lower fault energy [10, 13].
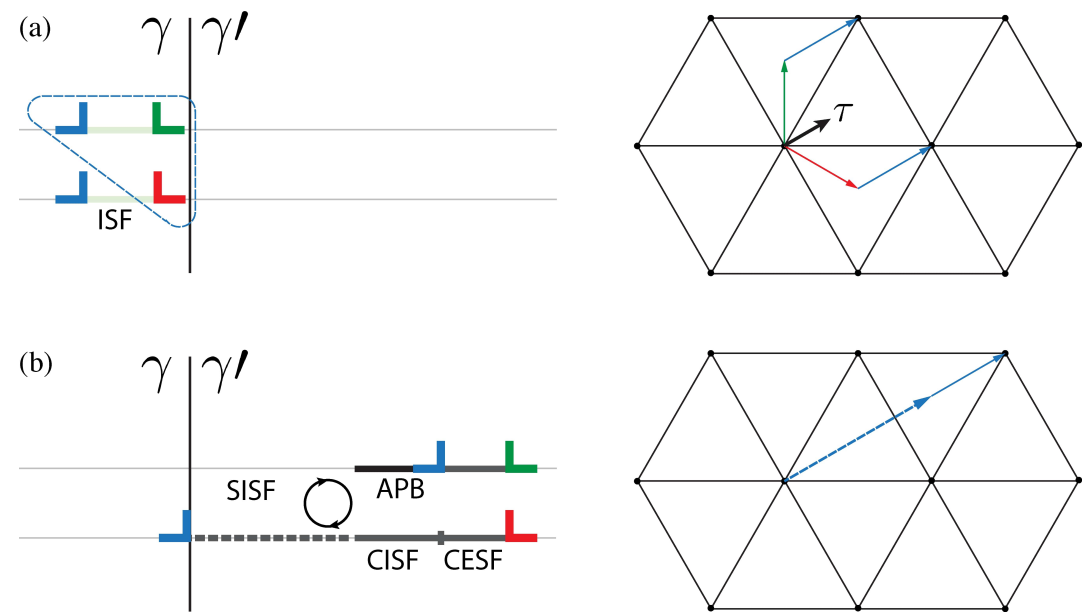

Figure 8: Dislocation structure of a $\operatorname{SISF}(\mathrm{a})$ before and (b) after shearing through the $\gamma$ ' precipitates. Each figure includes an edge-on view of the slip planes (left) and a top view of the Burgers vectors on a $\{111\}$ plane of the partials involved (right), where the atoms sit on the corners of the triangles. The arrows in (b) correspond to the circled superdislocation and the trailing partial in (a).

A slip plane with $\phi=30^{\circ}$ has two equally active slip systems with the same trailing partial, as shown in Figure 8(a). The angle between the Burgers vector of the two leading partials is $120^{\circ}$, meaning that they will attract each other. This construction gives the leading end of a SISF its extrinsic character observed experimentally. By moving together, this $\frac{a}{3}\langle 112\rangle$ superdislocation will have a total Burgers vector with the same orientation as the remaining $\frac{a}{6}\langle 112\rangle$ trailing partial and twice its magnitude. This is again the slip direction with the highest resolved shear stress, thus facilitating the viscous motion of the dislocations controlled by the diffusive processes of segregation and reordering.

The CESF formed constitutes a glide barrier upon forming at the interface but not once it enters the $\gamma$. The equilibrium distance between the partials bounding this fault $d_{C E S F}$ can be found with a similar derivation to that of equation (4). Considering both 
ends of the CESF to be stopped by a resisting frontal force, as well as a fully formed SESF,

$$
d_{C E S F}=\frac{A_{t}}{\gamma_{C E S F}-\gamma_{S I S F}+\frac{b}{2} \tau_{S}+\frac{b}{2 \sqrt{3}} \tau_{E}-F_{f 2}} .
$$

Condition $\mathrm{C}_{\mathrm{I}}$ for the leading superpartial to propagate through $\gamma /$ is

$$
\mathrm{C} 1_{\mathrm{I}}: 2 F_{a 2}-\gamma_{S I S F}-2 F_{f 1}-F_{f 2}>0,
$$

resulting in a SISF + ISF defect. Orowan looping of the trailing partial may also appear if

$$
\mathrm{C} 2_{\mathrm{I}}: F_{a 2}+\gamma_{I S F}-F_{f 2}>R_{1 c} .
$$

This would result in the formation of isolated SISFs within $\gamma^{\prime}$ precipitates. As both conditions have the same slope, a region where only the trailing partial is blocked will only appear if

$$
\gamma_{S I S F}+2 \gamma_{I S F}+2 F_{f 1}-F_{f 2}<2 R_{1 c}
$$

which is promoted by narrower $\gamma$ channels. A schematic diagram of conditions $\mathrm{C} 1_{\mathrm{I}}$ and $\mathrm{C} 2_{\text {I }}$ is shown in Figure 9(a). The fault formed will be that where the coordinates of the slip system within the domain of $\phi$ (from $-30^{\circ}$ to $30^{\circ}$ ) fall.

(a)

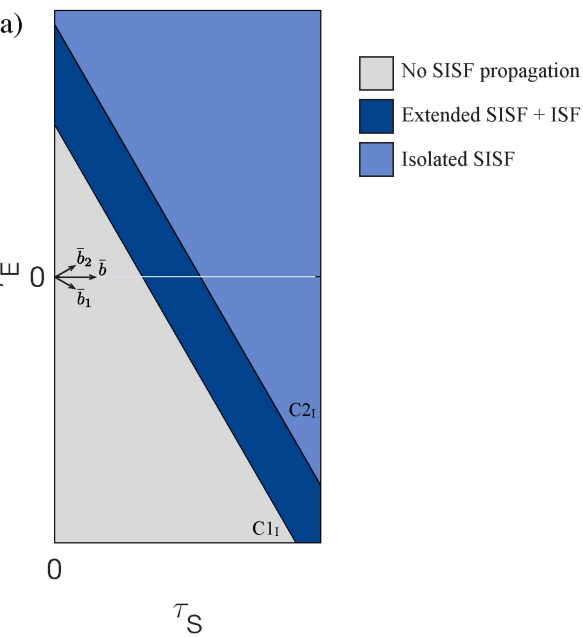

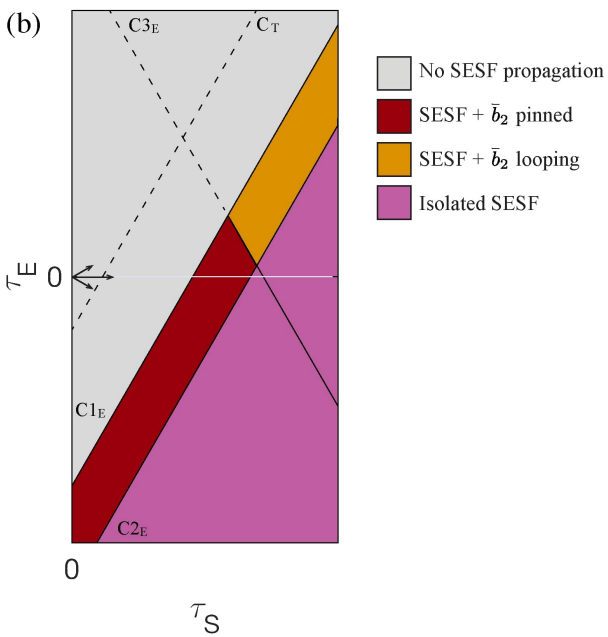

Figure 9: Schematic plot of the segregation assisted (a) SISF and (b) SESF shearing mechanisms with an aspect ratio of $1: 1$. The boundaries are labelled according to the conditions that form them.

The formation of stacking fault ribbons may only appear if the stress on the trailing partial is not large enough for athermal looping of the obstacles, appearing also in the extended SISF + ISF region. For this mechanism to occur, the planar fault undergoes the transition SISF-APB-SESF due to the passage of further $\frac{a}{6}<112>$ partials [16], with occurrence depending on the availability of suitable dislocations. In this scenario the trailing partial will shear the precipitate followed by an additional pair of dislocations that produce similar transformations in the planar faults, but this time forming a SESF 
rather than a SISF. Again the Burgers vectors of the superdislocation is the one with the highest Schmid factor in configurations with a high value of $\phi$.

\subsubsection{SESF-related mechanisms}

The dislocation structure of a SESF involves two partials with the same Burgers vector gliding through the precipitates on consecutive planes. Elastic interactions in this case make these partials glide separated from each other by a small distance with a complex intrinsic stacking fault (CISF) in-between. A SESF is then formed after glide of the second partial followed by local atomic reordering [9. Motion of both leading partials in this scenario is most favourable for orientations close to $\phi=30^{\circ}$.

The repulsive force between the leading partials with equal Burgers vectors results in a CISF of width

$$
d_{C I S F}=\frac{A_{t}}{\gamma_{C I S F}-\gamma_{S E S F}+\frac{b}{2} \tau_{S}-\frac{b}{2 \sqrt{3}} \tau_{E}-F_{f 1}} .
$$

Then, a condition $\mathrm{C}_{\mathrm{E}}$ for $\mathrm{SESF}$ propagation based on a force balance reads

$$
\mathrm{C} 1_{\mathrm{E}}: 2 F_{a 1}-\gamma_{S E S F}-2 F_{f 1}>0 .
$$

For $\phi=30^{\circ}$ there will be two coplanar slip systems sharing the same leading partial but with different trailing dislocations. As in the SISF analysis, the SESF might be originated by two dislocations with these Burgers vectors pushing against the $\gamma / \gamma^{\prime}$ interface in adjacent planes [44, as shown in Figure 10(a). There would be an attractive force between both trailing partials and their combined Burgers vector would equal that of each leading partial, as shown in Figure 10(b).
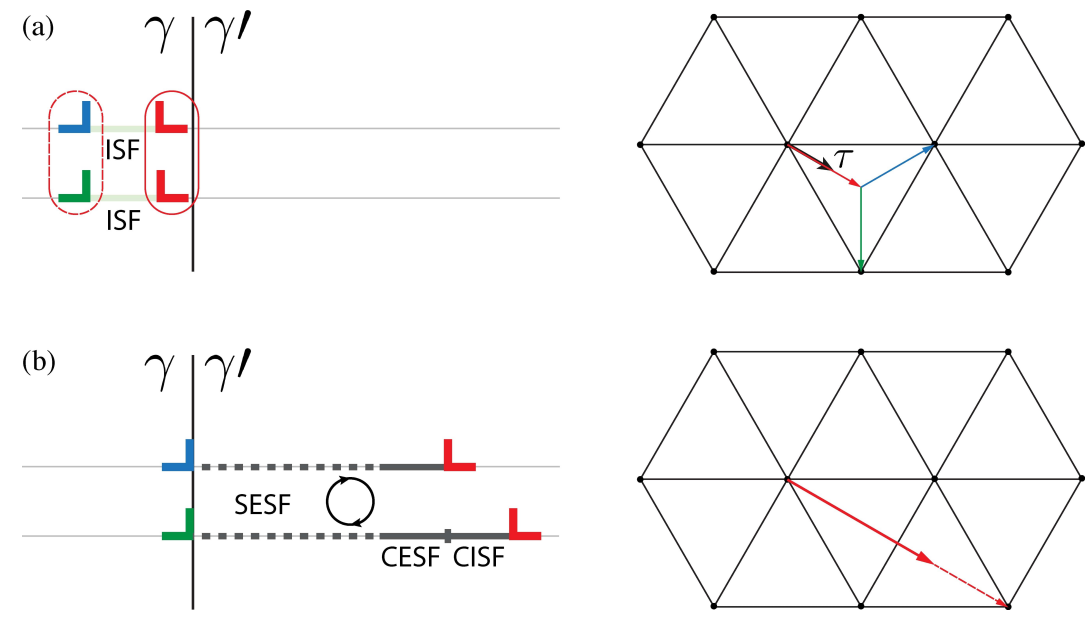

Figure 10: Dislocation structure of a SESF (a) before and (b) after shearing through the $\gamma^{\prime}$ precipitates. Each figure includes an edge-on view of the slip planes (left) and a top view of the Burgers vectors on a $\{111\}$ plane of the partials involved (right), where the atoms sit on the corners of the triangles. The arrows in (b) correspond to the circled dislocations in (a).

Upon decorrelation and glide of the leading ends, the trailing partials may evolve in three ways. Firstly, both of them could glide together in the matrix bypassing the 
precipitates via Orowan looping if

$$
\mathrm{C} 2_{\mathrm{E}}: F_{a 1}+\gamma_{E S F}-2 F_{f 2}>R_{1 c},
$$

resulting in isolated SESFs. Secondly, looping of a single partial while the other one remains pinned, converting the ESF in the matrix into an ISF with a similar energy penalty [45]. Note that the resulting dislocation structure around the precipitates would be identical to that experimentally observed by Décamps et al. [46] in a polycrystalline alloy. Kinetically this mechanism would occur if condition $\mathrm{C} 22_{\mathrm{E}}$ is false and

$$
\mathrm{C} 3_{\mathrm{E}}: F_{a 2}+\gamma_{E S F}-\gamma_{I S F}-F_{f 2}>R_{1 c} .
$$

This will happen only if one of the trailing partials experiences a higher force than the other one, which occurs for partial $\overline{b_{2}}$ as $\phi$ moves away from $-30^{\circ}$. These SESF conditions are schematically shown in Figure 9(b). A SESF/ISF alternating character would then appear if the coordinates of only one of the three coplanar slip systems lie in the $\overline{b_{2}}$ looping region. Thirdly, an extended extrinsic fault (SESF + ESF) would be promoted if both of these points are in the region of pinned trailing partials. Note that according to equations $(18)$ and $(19)$, the second and third options will only be possible for a material in which

$$
\gamma_{S E S F}+2 \gamma_{E S F}+2 F_{f 1}-4 F_{f 2}<R_{1 c}
$$

Lastly, segregation assisted microtwinning can be seen as a variation of the SESF mechanism in which more leading partials shear the matrix and the precipitates on adjacent planes. Different pathways for the order in which the planes are sheared have been proposed both in fcc and $\mathrm{L}_{2}$ structures [47] but the resulting stress orientation dependence is the same; glide on consecutive adjacent planes is considered here. It is not fully clear how microtwins nucleate, but the athermal stopping of the trailing partials may be a necessary condition. This would explain why this mechanism appears at lower stresses than SESFs [18, 21. Pinned trailing partials would give time for more dislocations to reach planes adjacent to the fault and increase the microtwin thickness as they thermally shear the precipitates (and athermally the matrix).

A leading partial gliding on a plane adjacent to a SESF would transform this fault into a 3-layer pseudo-twin, which after a series of local atomic reshuffling steps becomes a microtwin with two corresponding twin boundaries [9, 10. The force balance reads

$$
\mathrm{C}_{\mathrm{T}}: F_{a 1}+\gamma_{S E S F}-2 \gamma_{T p}-F_{f 1}>0
$$

where $\gamma_{T p}$ is the twin boundary energy in the precipitates. Simultaneously, the ESF in the matrix is replaced by two twin boundaries with energy $\gamma_{T m}$. Studies have shown that the energy difference between a 2- and a 3-layer fault is rather small both in the fcc and the $\mathrm{L}_{2}$ phases, i.e. $\gamma_{I S F} \approx \gamma_{E S F} \approx 2 \gamma_{T m}$ [48, 49] and $\gamma_{S E S F} \approx 2 \gamma_{T p}$ [47, 50]. The stress required to activate twinning from equation 22 is then expected to be low, as shown in Figure 9(b). Upon forming an extended SESF + ESF, microtwin thickening may be mostly limited by the diffusion and not by an additional stress requirement.

A summary of the thermal mechanisms described here is shown in Figure 11, together with the defects left by the partials when overcoming the precipitates. The thermally activated mechanisms should only occur if no athermal alternative is possible, as this allows the dislocations to push against the obstacles and drive the atomic diffusion process. 

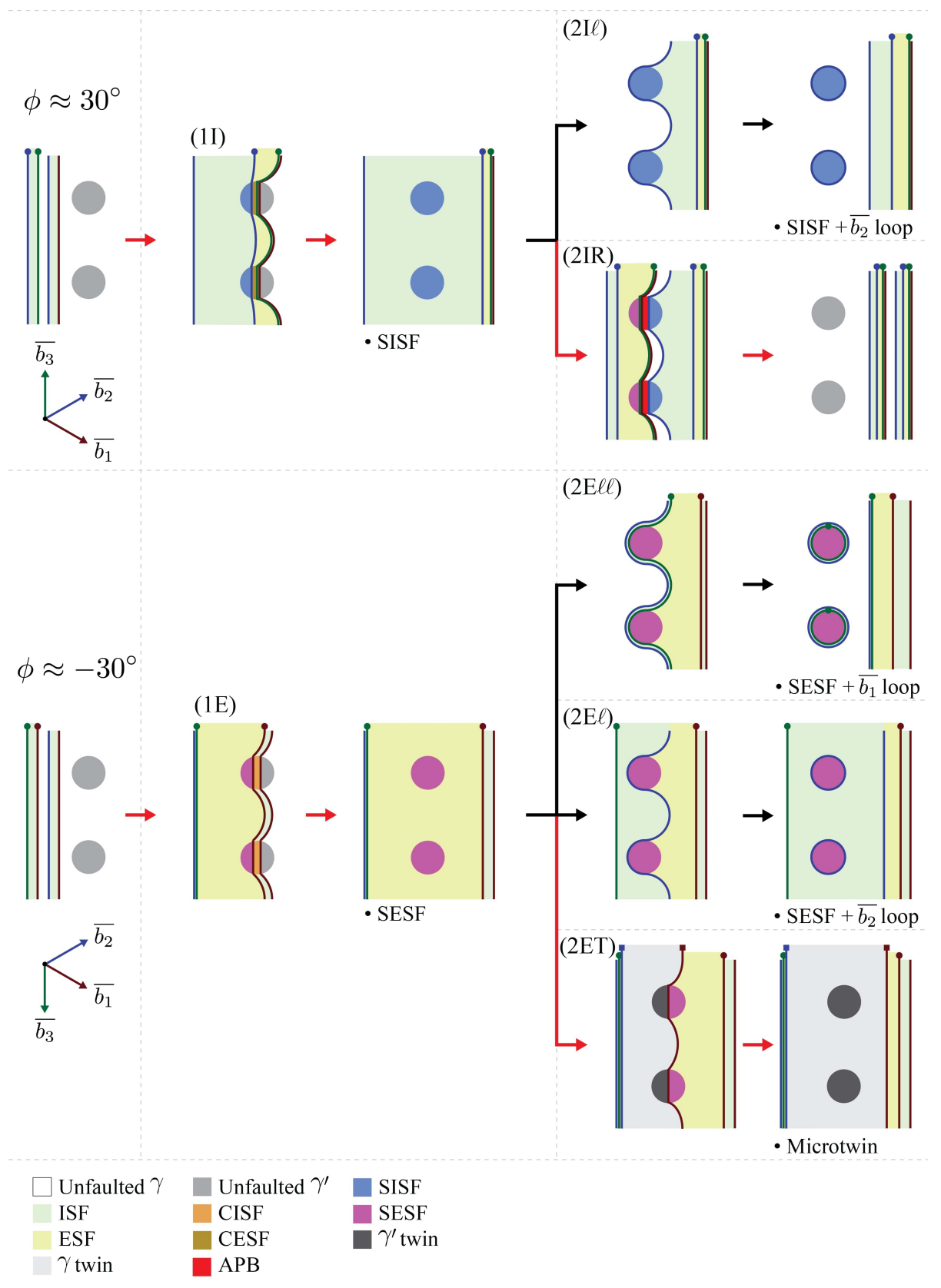

Figure 11: Schematic diagrams of the segregation assisted stacking fault shearing mechanisms driven by the glide of Shockley partials from left to right. A dot or square on top of a dislocation indicates that it is gliding on an adjacent plane. Black and red arrows denote the athermal or thermally-assisted nature of the mechanism, respectively. The defects left after the sweeping of each partial are annotated. 
A remaining question is: why do multiple types of faults appear during a single test? Looking at Figure 9 it is evident that the regions for the SISF and SESF mechanisms overlap in certain regions. These mechanisms can coexist as long as the appropriate dislocations meet at a $\gamma / \gamma$ ' interface. The models for both superlattice stacking faults share the $\overline{b_{1}}+\overline{b_{2}}$ dislocation, which in an orientation map for a single slip plane corresponds to the point with the highest Schmid factor. The other two coplanar slip systems are then the additional dislocation in each model; the one with the highest Escaig factor for the SISF and that with the lowest for the SESF. The combinations of these two pairs of points determine the faults that will be promoted. Figure 12 shows the superposition of the mechanistic maps for thermal and athermal mechanisms for an alloy with a $\gamma$ channel of $20 \mathrm{~nm}$, where the overlap of different mechanisms is denoted by the striped regions. Just from the geometry of the constraints it can be seen that SISF and SESF propagation will be promoted for positive and negative values of $\phi$, respectively.

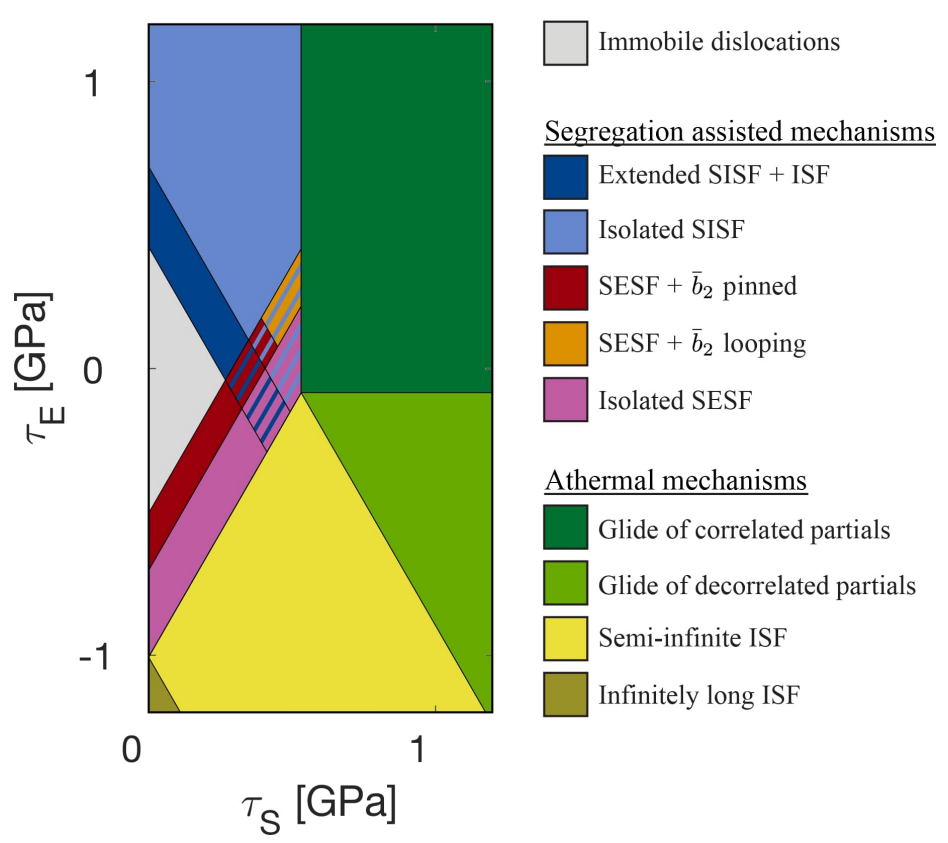

Figure 12: Regions for the athermal and segregation assisted mechanisms of precipitate bypassing in an alloy with cuboidal precipitates. The striped regions indicate that the mechanisms of both colours are operative. The values used for the calculation are $H=20 \mathrm{~nm}, \nu=0.33, \mu=58.6 \mathrm{GPa}, b=0.253$ $\mathrm{nm}, \alpha=1, \gamma_{I S F}=10 \mathrm{~mJ} / \mathrm{m}^{2}, \gamma_{E S F}=10 \mathrm{~mJ} / \mathrm{m}^{2}, \gamma_{A P B}=276 \mathrm{~mJ} / \mathrm{m}^{2}, \gamma_{C I S F}=196 \mathrm{~mJ} / \mathrm{m}^{2}$, $\gamma_{C E S F}=268 \mathrm{~mJ} / \mathrm{m}^{2}, \gamma_{S I S F}=62 \mathrm{~mJ} / \mathrm{m}^{2}, \gamma_{S E S F}=74 \mathrm{~mJ} / \mathrm{m}^{2}$.

\section{Discussion}

The analytical model for the athermal propagation of ISFs builds on a number of previous studies. Mechanistic maps like the one in Figure 7 have been generated before by simulations of individual partials for the case of Frank-Read sources [37, narrow 
channels [5, 19] and precipitates arrays [5, 20, 21, but the current analytical approach encompasses these to give a comprehensive prediction of dislocation behaviour. Moreover, the infinitely long stacking fault regime has never been described. Another implication of this analysis is that none of the conditions depend on the interaction force between the partials. Considering the anisotropic elasticity of the crystal would add another orientation dependence for the dissociation width [43, without any effect on the boundaries plotted.

An analysis of microstructures with multimodal precipitate size distributions may clarify the effects of secondary and tertiary precipitates on the stacking fault formation. Figure 13 shows a parametric analysis that considers the obstacle resistance forces for the corresponding defects via equations A.7 A.10), calculated with typical parameters of nickel-based superalloys: $\nu=0.33, \mu=58.6 \mathrm{GPa}, b=0.253 \mathrm{~nm}, f=0.1, \gamma_{I S F}=10$ $\mathrm{mJ} / \mathrm{m}^{2}$ 39, $\gamma_{A P B}=276 \mathrm{~mJ} / \mathrm{m}^{2}$ and $\gamma_{C I S F}=196 \mathrm{~mJ} / \mathrm{m}^{2}$ [45, with $\theta=90^{\circ}, \alpha=1$ and $\xi=1$. There is a transition from shearing to looping for the leading partial similar to that of perfect dislocations 51 at a radius $r=r_{c}$. The trailing partial experiences a very small resistance in the former regime because the energy of a CISF is very similar to that of an APB [42, 52, promoting further shearing. However, larger precipitates are bypassed via looping of both partials, which generates the large peak in $R_{2}$ seen in the plot. A combination of these mechanisms is expected accounting for the variability in precipitate sizes and spacings. Yet, large secondary precipitates bypassed via Orowan looping promote the formation of athermal stacking faults, whereas smaller tertiaries are only sheared.

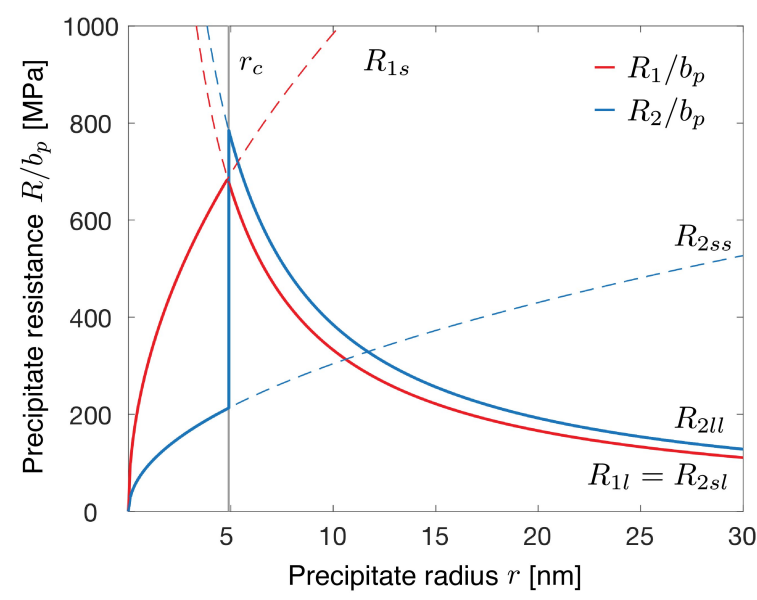

Figure 13: Precipitate resistance $R / b_{p}$ for the leading and trailing partial dislocations as a function of mean particle size. The solid lines denote the actual resistance and the dashed lines represent the individual mechanisms. The transition $r_{c}$ from shearing to looping for the leading partial is marked for reference.

In a more general context, consider now the angle

$$
\theta_{P}=-\arctan \left(\frac{\sqrt{3}\left(2 \gamma_{I S F}+R_{1}-R_{2}+F_{f 1}-F_{f 2}\right)}{R_{1}+R_{2}+F_{f 1}+F_{f 2}}\right)
$$


between the horizontal axis and a line from the origin to point $P$ in Figure 7. This can be interpreted as the parameter determining the likelihood for an fcc crystal to develop athermal stacking faults compared to perfect dislocation glide. If this is lower than $-30^{\circ}$, then it is impossible to promote stacking fault propagation without first reaching the glide of correlated partials regime in a coplanar slip system. This conclusion arises by considering the constraints regarding the stress state in coplanar slip systems [22]. The minimum resolved shear stress for stacking fault propagation in this condition is then that of point $P$. Inspection of equation (23) shows that the key parameters to promote ISF propagation are a low stacking fault energy and a high trailing partial glide resistance $R_{2}+F_{f 2}$, which is in agreement with the parametric analysis in Figure 13

The analysis on the different configurations of superlattice stacking faults paves the way towards a sound understanding of the dislocation pathways to accommodate plastic deformation in this creep regime. From experimental evidence in the literature and in the current work, it is clear that there is a strong stress orientation dependence behind the formation and propagation of these faults. Equations (13) and (17) for the complex stacking faults widths, calculated with $\gamma_{C E S F}=268 \mathrm{~mJ} / \mathrm{m}^{2}, \gamma_{S I S F}=62 \mathrm{~mJ} / \mathrm{m}^{2}$ [45], $\gamma_{S E S F}=74 \mathrm{~mJ} / \mathrm{m}^{2}[45]$ and the stress attained in a high resolution TEM study $\left(\tau_{S}=343\right.$ MPa and $\tau_{E}=149 \mathrm{MPa}$ ) [13, result in $d_{C E S F}=1.05 \mathrm{~nm}$ and $d_{C I S F}=1.76 \mathrm{~nm}$. These values are $19 \%$ lower and $37 \%$ higher than the experimental measurements, respectively. With all the assumptions made these results are reassuringly accurate, which validates the simple force balances employed as a first approach.

Due to geometric constraints on the slip systems, the angle $\phi$ can effectively capture the transition from intrinsic to extrinsic faults due to the different partials experiencing dissimilar shear stresses 22. The mechanistic maps from Figure 12 can be condensed into those in Figure 14, which cover all the possible shear stresses and orientations that a single slip plane may experience. Here these are plotted for different channel widths to showcase the large effect of the microstructure morphology, showing that an increase in the channel width promotes the formation of isolated faults. This occurs, according to equations (16) and (21), because athermal dislocation glide along the matrix becomes easier and the trailing partials are no longer pinned. Simultaneously, athermal perfect dislocation glide becomes easier for the same reason. It must be emphasised that all the predictions here refer to the critical channel width and not its mean value, as it only takes a few pinning points to hold back the dislocation. Also note that there is a difference in reading the appearance of athermal and thermally assisted faults from the mechanistic maps. The latter rely on the stresses of two coplanar $\frac{a}{2}\langle 110\rangle$ slip systems, which can be extracted uniquely from the region within the domain of the angle $\phi$, as shown in Figure 14. The athermal faults are instead formed from individual perfect dislocations, so their appearance is better understood from a full map as that in Figure 7.

It is possible to translate these results for specific loading modes by projecting the mechanistic maps onto the plot of the loading axis on the stereographic triangle for the most highly stressed slip plane. Figures 15 (a) and 15(b) accomplish this for the case of uniaxial tension and compression by accounting for the shear stress on the primary slip plane for every possible loading axis. The inserts on the top left correspond to the range of Schmid and Escaig stresses that may be attained for each load configuration, and effectively map these coordinate systems onto the crystal stereographic projection. While the maps in Figure 14 are very valuable due to their universality, the equivalent in the inverse pole figures facilitates a direct comparison between models and experiments. 
(a)

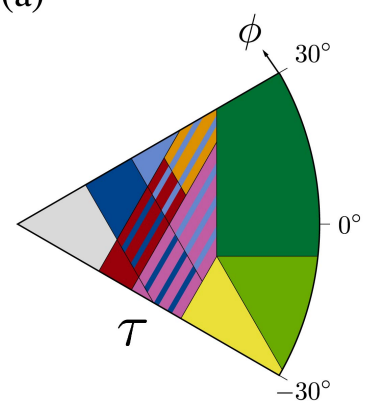

Narrow channel (b)

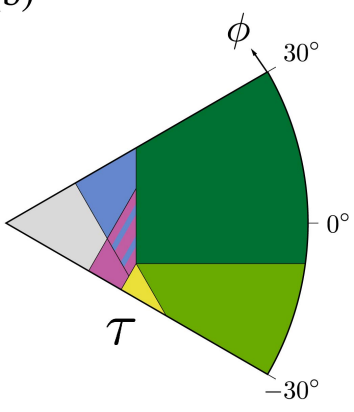

Wide channel
Immobile dislocations

Segregation assisted mechanisms Extended SISF + ISF / SF ribbon

Isolated SISF

Extended SESF + ESF / Microtwinning

Extended SESF + ISF

Isolated SESF

Athermal mechanisms

Glide of correlated partial

Glide of decorrelated partials

Semi-infinite ISF

Infinitely long ISF

Figure 14: Stress-orientation maps of athermal and segregation assisted precipitate bypassing mechanisms for alloys with (a) narrow or (b) wide $\gamma$ channels. The striped regions indicate that the mechanisms of both colours are operative.

Whilst these plots do not account for the microstructure variability, they give an idea of how the regions in the $\phi$ maps translate to real crystal orientations and stresses.

(a)

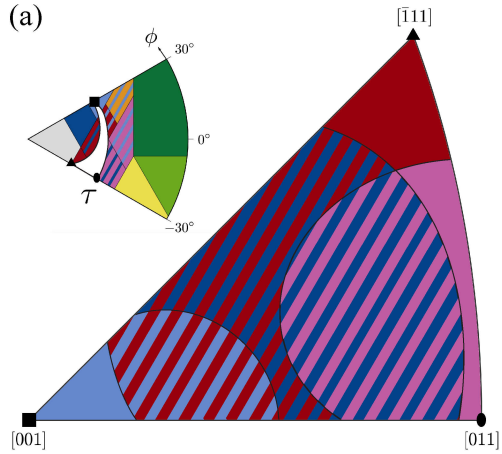

(b)

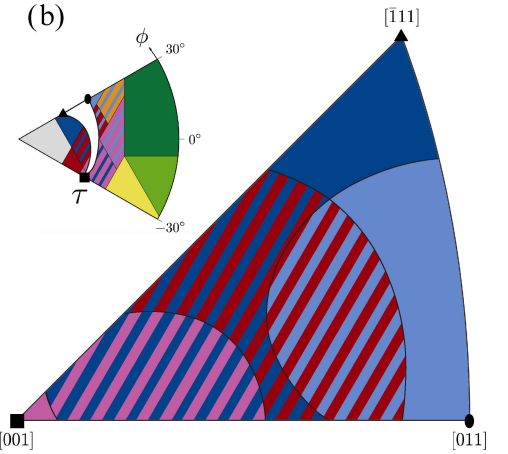

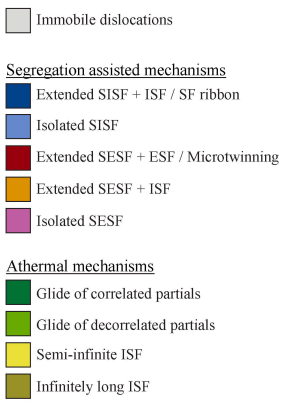

Figure 15: Inverse pole figures for (a) uniaxial tension and (b) uniaxial compression with the predicted stacking fault mechanisms, where the white curved triangles in the inserts on the top left represent the stresses attained by the slip plane with the highest stress for all possible orientations. The values used for the calculations correspond to those used to plot Figure 14 (a) and an axial stress of $800 \mathrm{MPa}$.

Data from the literature for single crystals in Figure 1(b) seems to match the analytically obtained orientation dependence. Whilst these tests were performed with different alloys, temperatures and applied stresses, the overall tendencies are successfully reproduced.

Grains with different orientations in the polycrystalline nickel-based superalloy studied here show broadly similar trends, as seen by comparing Figure 15(a) with Figure 4. While SISFs appear in most grains, SESFs clearly increase in numbers as the tensile axis moves away from a $\langle 001\rangle$ direction. In addition, more isolated faults are observed near the left and right edges of the inverse pole figure, whereas extended configurations appear more towards the centre, in agreement with the predictions of the model.

There is clearly more scatter in the types of faults found in each grain compared 
to observations in single crystals. One of the reasons for this is the large variability of precipitate sizes and $\gamma$-channel widths, specially for the flowery secondary $\gamma^{\prime}$. This presents each dislocation with a series of obstacles with different resistances along the glide plane that may promote a change in the deformation mechanism, e.g. the observed isolated SESFs that then turn into extended SESFs+ESF upon stopping of the trailing partials.

Another factor responsible for the deviations from the predicted behaviour in polycrystals must be the need for strain compatibility between neighbouring grains. Consider for example grain 1 loaded close to a $\langle\overline{1} 11\rangle$ direction, which has a low Schmid factor and a high axial stiffness 53 making it harder to deform compared to its neighbouring grains. Due to load partitioning the shear stresses in this grain may not only increase in magnitude, as hinted by the observed larger dislocation density, but also rotate to promote more SISFs. These incompatibility effects must also affect other grains but to a lesser degree. Whilst the more complex stress states developed in polycrystalline alloys makes them harder to investigate, this study shows that the stress orientation analysis is effective at capturing the deformation behaviour even for these small plastic strains. Incorporation of the introduced stress and orientation dependencies into crystal plasticity models may elucidate more effects related to the interactions between grains during creep of polycrystalline alloys.

A further novel finding of the current study is the observation of a SISF + ESF configuration, for which we propose the mechanism presented in Figure16. Consider the formation of an ISF in the matrix with pinning of the $\overline{b_{2}}$ trailing partial and SISFs in the precipitates driven by a $\overline{b_{1}}$ partial and a $\overline{b_{3}}+\overline{b_{2}}$ perfect dislocation, i.e. mechanism (1I) in Figure 11. If an additional $\overline{b_{1}}$ leading partial glides on a plane adjacent to the ISF, forming an ESF in the matrix, it will be impeded from shearing a precipitate as this would transform the SISF into a complex defect with an energy closer to that of an APB. Instead, athermal Orowan bowing would result in SISFs with $\overline{b_{1}}$ loops around them and an ESF in the matrix, as long as the trailing partial of the new dislocation is also pinned. The stress requirements for this mechanism are those needed for the SISF + ISF configuration, in addition to condition

$$
\mathrm{C} 3_{\mathrm{I}}: F_{a 1}+\gamma_{I S F}-\gamma_{E S F}-F_{f 1}>R_{1 c},
$$

analogous to equation (20). This mechanism would then be promoted in a region in Figure 14 similar to that of the extended SESF + ISF but mirrored along the $\tau_{S}$ axis, for $\phi \sim 0^{\circ}$. Further investigations into this new type of fault are necessary to validate this mechanism.

The combined Burgers vector of all the partials involved in the mechanisms analysed are drawn in Figure 17, regardless of whether the faults develop over one or more adjacent planes. While all the equations developed in this study show in the mechanistic maps as lines perpendicular to the Burgers vectors of either the leading or the trailing partial, the regions where each mechanism appears are consistent with the orientation of the combined Burgers vector. The only exception are the extended faults with alternating character, which appear switched in Figure 12 because the athermal looping of precipitates requires a higher stress than the formation of the superlattice faults. In the case of larger channels, as those in this alloy, the boundaries from equations $(20)$ and $(24)$ shift these regions closer to $\phi \sim 0^{\circ}$, where both of these faults are found experimentally. 


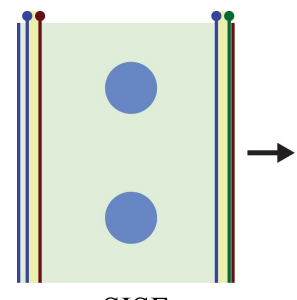

- SISF

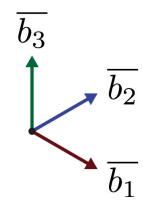

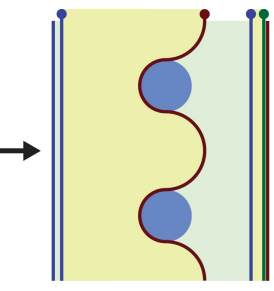

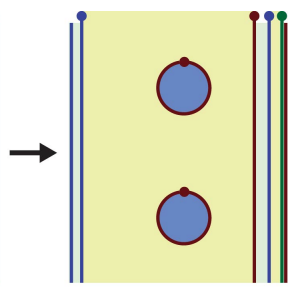

- $\mathrm{SISF}+\overline{b_{1}}$ loop

Figure 16: Sequence of the proposed mechanism for a SISF + ESF configuration. From a SISF + ISF configuration, a $\overline{b_{1}}$ partial glides on an adjacent plane looping around the precipitates converting the ISF into an ESF.

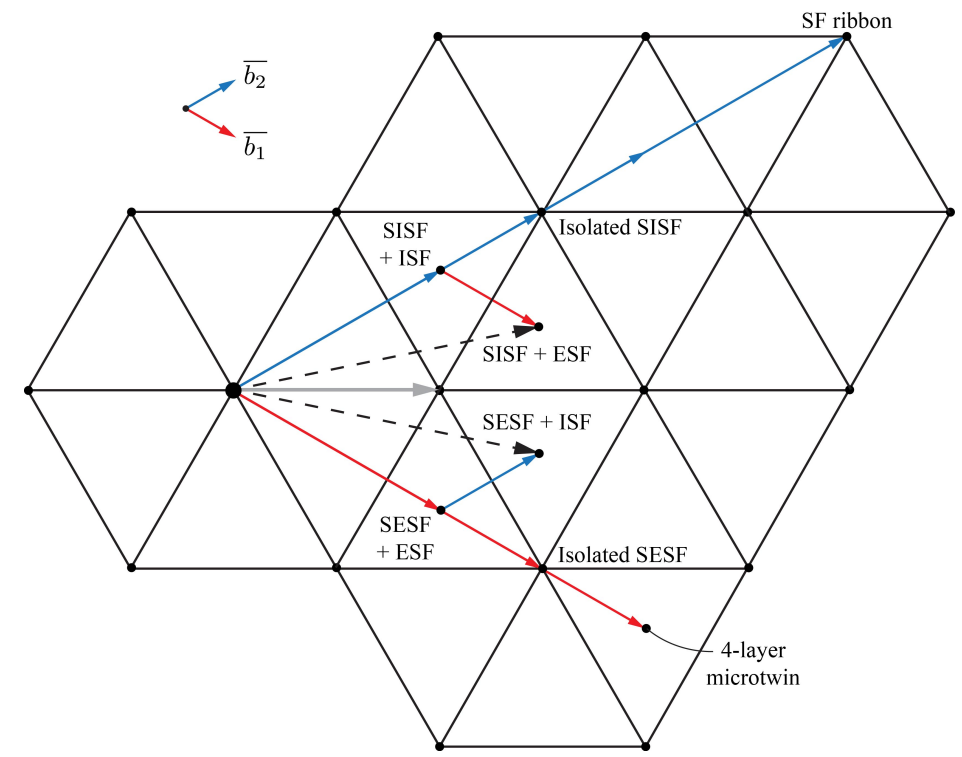

Figure 17: Schematic diagram of a $\{111\}$ plane with arrows that represent the combined Burgers vector of all the partials that glide over multiple adjacent slip planes for each mechanism. The dashed arrows represent the stacking faults with alternating character and the grey arrow the Burgers vector of a perfect dislocation.

This general picture of the superlattice faults in nickel-based superalloys offers an overview of how crystals find intricate ways to accommodate deformation in the direction of the shear stress. While the detailed scrutiny of the shear stress in the slip plane may be enough to analyse the propagation of these defects, nucleation remains unclear. Further studies in this subject are required to elucidate the mechanisms by which dislocations with different Burgers vectors end up on adjacent planes pushing against the precipitates, 
possibly via cross slip from the dislocation tangles near the grain boundaries.

\section{Conclusions}

This work develops the first ever comprehensive theory of stress, orientation and microstructure dependence for the appearance of planar faults in nickel-based superalloys. The main findings are:

- Athermal stacking fault configurations depend mainly on the microstructure of the material, whereas thermally assisted superlattice faults have a larger dependence on stress orientation.

- There is a good qualitative agreement between this model and results from the literature for single crystals. Larger deviations exist in polycrystals likely due to strain compatibility phenomena and the variability in precipitate morphologies.

- A new extended superlattice stacking fault configuration is identified for the first time, consisting of an ESF with SISFs in the precipitates. A dislocation pathway to produce such fault is proposed, although further experimental validation is required.

\section{Acknowledgements}

F.D. León-Cázares is grateful for funding from CONACyT and the Cambridge Trust. This work was also supported by Rolls-Royce plc and the Engineering and Physical Sciences Research Council (EPSRC) under the Strategic Partnership, Grant Numbers $\mathrm{EP} / \mathrm{H} 022309 / 1$ and $\mathrm{EP} / \mathrm{H} 500375 / 1$.

\section{Appendix A. Obstacle surpassing mechanisms}

Obstacle surpassing mechanisms depend on the line tension $T$ of the dislocation as the radius of curvature $\varrho$ it will adopt is inversely proportional to the force applied $F$ through the relation $\varrho=T / F$. This tension, which can be thought of as the self-energy per unit distance of the dislocation, is proportional to $G b^{2}$, but it also depends on the character and even on whether it belongs to a perfect dislocation or is a completely isolated partial 39. Modelling of this parameter has been performed before in a more complete way [39], although for simplicity it is here assumed to be $T=\alpha G b^{2} / 2$ in agreement with other approaches in the literature, where $\alpha$ is a dimensionless parameter close to one.

In microstructures with large cuboidal precipitates (Figure A.18(a)) the antiphase boundary energy restricts most of the deformation to the channels in-between. The required stress for a dislocation to enter a channel of width $H$ is $\tau=2 T / H b$, which in the current framework becomes a resistance for the leading and trailing partials of

$$
R_{1 c}=\frac{\alpha G b_{p}^{2}}{H}
$$

and

$$
R_{2 c}=\frac{\alpha G b_{p}^{2}}{\frac{H-2 d_{c}}{25}},
$$



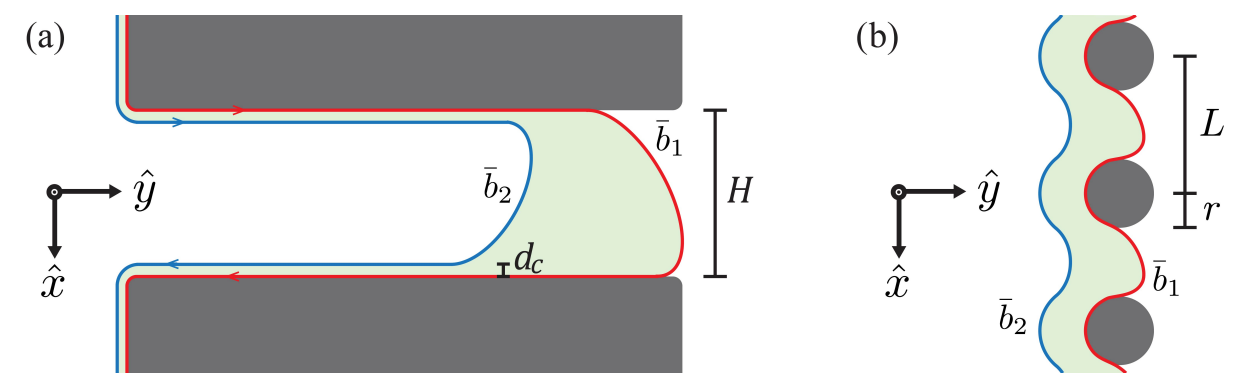

Figure A.18: Schematic diagram of dislocation glide (a) through a $\gamma$ channel and (b) pushing against an array of spherical precipitates.

respectively, where $d_{c}$ is the stacking fault width in the $\hat{x}$-direction.

The trailing partial needs to overcome a larger resistance due to the repulsion force from the segments left by the leading partial pushing against the obstacles. Assuming that both dislocations are already decorrelated and far from each other, the trailing partial will start gliding through the channel when

$$
F_{a 2}+\gamma_{I S F}-F_{f 2}=R_{2 c}
$$

Simultaneously, the force balance in the $\hat{x}$-direction on a straight segment of one of the trailing partials reads

$$
F_{a 2}+\gamma_{I S F}-F_{f 2}-\frac{A}{d_{c}}-\frac{A_{t}}{H-2 d_{c}}-\frac{A}{H-d_{c}}=0,
$$

where $A_{t} / x$ is the interaction force between two parallel dislocations separated by a distance $x$ on the same slip plane and with the same Burgers vector, and

$$
A_{t}=\frac{4-2 \nu-2 \nu \sin \left(\pi / 6+2 \theta_{2}\right)}{8 \pi(1-\nu)} G b_{p}^{2}
$$

Note that both $A$ and $A_{t}$ in equation A.4 must be obtained for a dislocation character $\theta_{2}=\theta+\pi / 2$ because these segments are perpendicular to the moving end of the partials. Rearranging and solving equations A.2, (A.3) and A.4 gives two solutions, only one of which has a physical meaning:

$$
\begin{gathered}
d_{C}=\frac{H}{-4+8 \pi(1-\nu)+2 \nu+2 \nu \sin \left(\pi / 6+2 \theta_{2}\right)}\left(4 \pi(1-\nu)-2 \nu \cos \left(2 \theta_{2}\right)+\nu \sin \left(\pi / 6+2 \theta_{2}\right)\right. \\
\left.-\sqrt{16 \pi^{2}(1-\nu)^{2}+2(2-\nu)^{2}-8 \pi\left(2-3 \nu+\nu^{2}\right)-4(2-\nu) \nu \cos \left(2 \theta_{2}\right)+4 \nu^{2} \cos \left(2 \theta_{2}\right)^{2}-(4-8 \pi(1-\nu)-2 \nu) \nu \sin \left(\pi / 6+2 \theta_{2}\right)+\nu^{2} \sin \left(\pi / 6+2 \theta_{2}\right)^{2}}\right) .
\end{gathered}
$$

This means that $d_{c}$ is proportional to $H$ by a factor that only depends on $\nu$ and $\theta_{2}$, and ranges between 0.07 and 0.139 for a typical value of $\nu=0.3$.

The analysis of an array of spherical obstacles (Figure 13(b)) constitutes a challenge due to the variability in the morphology and surroundings of the precipitates. Classical models of precipitate strengthening [51, 54, 55] are applied here to the individual partials 
considering that the promoted mechanism is that with the lowest critical resolved shear stress.

The force balance at the point of maximum particle resistance during shearing of a precipitate cross section with an energy penalty of $\gamma_{P}$ by a dislocation with Burgers vector $b$ leads to a general resistance

$$
R_{s}=\frac{2 r}{\lambda\left(b, \gamma_{P}\right)} \gamma_{P}
$$

where $r$ is the mean precipitate radius,

$$
\lambda\left(b, \gamma_{P}\right)=\left(\frac{\alpha G}{2 \gamma_{P} r}\right)^{1 / 2} \xi b L
$$

the Friedel sampling length of the dislocation [56], $\xi$ a correction parameter and $L=$ $(2 \pi / 3 f)^{1 / 2} r$ the mean precipitate spacing [57. Equations A.7 and A.8 can be used to calculate the forces $R_{1 s}$ and $R_{2 s s}$. These Friedel statistics capture the effect of the microstructure variability on the effective precipitate spacing $\lambda$. This parameter has been analysed in detail in multiple 2D simulations by looking at the evolution of one dislocation gliding along a material with discrete obstacles. This is also the way in which single Shockley partials evolve when fully decorrelated, as out-of-plane glide and pileups are not an option. Deviations from equation A.8 have been systematically studied for different precipitate strengths 58 and ranges of volume fractions 59, all of which are incorporated into $\xi$. The reader is referred to reference [56] for further details and numerical evaluations of this parameter.

Alternatively, Orowan looping is analogous to glide through $\gamma$ channels. The obstacle force for a leading partial is given by

$$
R_{1 \ell}=\frac{\alpha G b_{p}^{2}}{L-2 r} .
$$

A variation introduced by Unocic et al. [5] considers the elastic contribution of the trailing dislocation pushing via the interaction force that results in a curved boundary in Section 4.1. These effects are ignored here for simplicity, but adding them is straightforward. Then, the force required for a trailing partial to loop a precipitate with a $\overline{b_{1}}$ loop around it is

$$
R_{2 \ell \ell}=\frac{\alpha G b_{p}^{2}}{L-2 r-2 d_{\ell \ell}},
$$

where $d_{\ell \ell}$ can be found by substituting $H$ with $(L-2 r)$ in equation (A.6). Alternatively, the trailing partial may induce the required stress for the loop to collapse and annihilate by shearing the precipitate. Due to the more complicated geometry of the dislocation configuration, a proper analysis would require a discretised line tension approach.

\section{References}

[1] F. León-Cázares, R. Schlütter, T. Jackson, E. Galindo-Nava, C. Rae, A multiscale study on the morphology and evolution of slip bands in a nickel-based superalloy during low cycle fatigue Acta Materialia 182 (2020) 47-59. 
[2] P. M. Anderson, J. P. Hirth, J. Lothe, Theory of dislocations, 3rd Edition, Cambridge University Press, New York, 2017.

[3] A. G. Jackson, Handbook of Crystallography For Electron Microscopists and Others, 1st Edition, Springer-Verlag, New York, 1991.

[4] N. Thompson, Dislocation Nodes in Face-Centred Cubic Lattices Proceedings of the Physical Society. Section B 66 (6) (1953) 481-492.

[5] R. R. Unocic, N. Zhou, L. Kovarik, C. Shen, Y. Wang, M. J. Mills, Dislocation decorrelation and relationship to deformation microtwins during creep of a $\gamma^{\prime}$ precipitate strengthened Ni-based superalloy, Acta Materialia 59 (2011) 7325-7339.

6] B. C. De Cooman, Y. Estrin, S. K. Kim, Twinning-induced plasticity (TWIP) steels Acta Materialia 142 (2018) 283-362.

7] D. Barba, T. M. Smith, J. Miao, M. J. Mills, R. C. Reed, Segregation-Assisted Plasticity in Ni-Based Superalloys Metallurgical and Materials Transactions A 49 (9) (2018) 4173-4185.

[8] A. Breidi, J. Allen, A. Mottura, First-principles modeling of superlattice intrinsic stacking fault energies in Ni3Al based alloys Acta Materialia 145 (2018) 97-108.

[9] M. Kolbe, The high temperature decrease of the critical resolved shear stress in nickel-base superalloys Materials Science and Engineering: A 319-321 (2001) 383-387.

[10] L. Kovarik, R. Unocic, J. Li, P. Sarosi, C. Shen, Y. Wang, M. Mills, Microtwinning and other shearing mechanisms at intermediate temperatures in Ni-based superalloys Progress in Materials Science 54 (6) (2009) 839-873.

[11] T. M. Smith, R. R. Unocic, H. Deutchman, M. J. Mills, Creep deformation mechanism mapping in nickel base disk superalloys Materials at High Temperatures 33 (4-5) (2016) 372-383.

[12] G. Viswanathan, R. Shi, A. Genc, V. Vorontsov, L. Kovarik, C. Rae, M. Mills, Segregation at stacking faults within the $\gamma^{\prime}$ phase of two Ni-base superalloys following intermediate temperature creep Scripta Materialia 94 (2015) 5-8.

[13] V. Vorontsov, L. Kovarik, M. Mills, C. Rae, High-resolution electron microscopy of dislocation ribbons in a CMSX-4 superalloy single crystal, Acta Materialia 60 (12) (2012) 4866-4878.

[14] R. Unocic, G. Viswanathan, P. Sarosi, S. Karthikeyan, J. Li, M. Mills, Mechanisms of creep deformation in polycrystalline Ni-base disk superalloys Materials Science and Engineering: A 483-484 (1-2 C) (2008) 25-32.

[15] N. Matan, D. Cox, P. Carter, M. Rist, C. Rae, R. Reed, Creep of CMSX-4 superalloy single crystals: effects of misorientation and temperature Acta Materialia 47 (5) (1999) 1549-1563.

[16] C. Rae, R. Reed, Primary creep in single crystal superalloys: Origins, mechanisms and effects Acta Materialia 55 (3) (2007) 1067-1081.

[17] G. Viswanathan, P. Sarosi, M. Henry, D. Whitis, W. Milligan, M. Mills, Investigation of creep deformation mechanisms at intermediate temperatures in René 88 DT Acta Materialia 53 (10) (2005) 3041-3057.

[18] D. Barba, E. Alabort, S. Pedrazzini, D. Collins, A. Wilkinson, P. Bagot, M. Moody, C. Atkinson, A. Jérusalem, R. Reed, On the microtwinning mechanism in a single crystal superalloy Acta Materialia 135 (2017) 314-329.

[19] J. Douin, F. Pettinari-Sturmel, A. Coujou, Dissociated dislocations in confined plasticity, Acta Materialia 55 (19) (2007) 6453-6458.

[20] H. Deutchman, P. Phillips, N. Zhou, M. Samal, S. Ghosh, Y. Wang, M. Mills, Deformation Mechanisms Coupled with Phase Field and Crystal Plasticity Modeling in a High-Temperature Polycrystalline Ni-Based Superalloy, in: Superalloys 2012, Proceedings of the International Symposium on Superalloys, TMS, 2012, pp. 25-33.

[21] T. M. Smith, L. V. Duchao, T. Hanlon, A. Wessman, Y. Wang, M. J. Mills, Determination of Orientation and Alloying Effects on Creep Response and Deformation Mechanisms in Single Crystals of Ni-Base Disk Superalloys, in: Superalloys 2016, Proceedings of the International Symposium on Superalloys, TMS, 2016, pp. 579-588.

[22] F. D. León-Cázares, C. M. F. Rae, A Stress Orientation Analysis Framework for Dislocation Glide in Face-Centred Cubic Metals, Crystals 10 (6) (2020) 445.

[23] D. Barba, E. Alabort, D. Garcia-Gonzalez, J. Moverare, R. Reed, A. Jérusalem, A thermodynamically consistent constitutive model for diffusion-assisted plasticity in Ni-based superalloys International Journal of Plasticity 105 (2018) 74-98.

[24] B. Décamps, A. J. Morton, M. Condat, On the mechanism of shear of $\gamma^{\prime}$ precipitates by single $(\mathrm{a} / 2)<110>$ dissociated matrix dislocations in Ni-based superalloys Philosophical Magazine A 64 (3) (1991) 641-668.

[25] B. Décamps, J.-M. Pénisson, M. Condat, L. Guétaz, A. J. Morton, High resolution imaging of 
shearing configurations of $\gamma^{\prime}$ precipitates in Ni-based superalloys, Scripta Metallurgica et Materialia 30 (11) (1994) 1425-1430.

[26] M. Benyoucef, A. Coujou, F. Pettinari-Sturmel, S. Raujol, B. Boubker, N. Clément, Dynamics of micromechanisms controlling the mechanical behaviour of industrial single crystal superalloys Sadhana 28 (1-2) (2003) 129-146.

[27] D. Knowles, Q. Chen, Superlattice stacking fault formation and twinning during creep in $\gamma / \gamma$ 'single crystal superalloy CMSX-4, Materials Science and Engineering: A 340 (1-2) (2003) 88-102.

[28] N. Tsuno, S. Shimabayashi, K. Kakehi, C. M. F. Rae, R. C. Reed, Tension/Compression asymmetry in yield and creep strengths of Ni-based superalloys, in: Superalloys 2008, Proceedings of the International Symposium on Superalloys, 2008, pp. 433-442.

[29] T. Smith, Y. Rao, Y. Wang, M. Ghazisaeidi, M. Mills, Diffusion processes during creep at intermediate temperatures in a Ni-based superalloy. Acta Materialia 141 (2017) 261-272.

[30] M. Hardy, R. Reed, D. Crudden, A nickel-base superalloy. EP3112485B1 (2018).

[31] D. B. Williams, C. B. Carter, Transmission Electron Microscopy: A Textbook for Materials Science. Part 3: Imaging, 2nd Edition, Springer, New York, 2009.

[32] F. Di Gioacchino, J. Quinta da Fonseca, Plastic Strain Mapping with Sub-micron Resolution Using Digital Image Correlation, Experimental Mechanics 53 (5) (2013) 743-754.

[33] F. Bachmann, R. Hielscher, H. Schaeben, Texture Analysis with MTEX - Free and Open Source Software Toolbox, Solid State Phenomena 160 (2010) 63-68.

[34] R. Hielscher, C. B. Silbermann, E. Schmidl, J. Ihlemann, Denoising of crystal orientation maps Journal of Applied Crystallography 52 (5) (2019) 984-996.

[35] J.-B. Baudouin, G. Monnet, M. Perez, C. Domain, A. Nomoto, Effect of the applied stress and the friction stress on the dislocation dissociation in face centered cubic metals Materials Letters 97 (2013) 93-96.

[36] N. Burbery, R. Das, G. Ferguson, Mobility of dissociated mixed dislocations under an Escaig stress Modelling and Simulation in Materials Science and Engineering 25 (4) (2017) 045001.

[37] M. de Koning, W. Cai, V. V. Bulatov, Anomalous Dislocation Multiplication in FCC Metals Physical Review Letters 91 (2) (2003) 025503.

[38] P. Franciosi, A. Zaoui, Multislip in f.c.c. crystals a theoretical approach compared with experimental data, Acta Metallurgica 30 (8) (1982) 1627-1637.

[39] S. Raujol, M. Benyoucef, D. Locq, P. Caron, F. Pettinari, N. Clement, A. Coujou, Decorrelated movements of Shockley partial dislocations in the $\gamma$-phase channels of nickel-based superalloys at intermediate temperature, Philosophical Magazine 86 (9) (2006) 1189-1200.

[40] B. Li, M. Sui, S. Mao, Twinnability Predication for fcc Metals, Journal of Materials Science \& Technology 27 (2) (2011) 97-100.

[41] A. Hunter, R. F. Zhang, I. J. Beyerlein, T. C. Germann, M. Koslowski, Dependence of equilibrium stacking fault width in fcc metals on the $\gamma$-surface Modelling and Simulation in Materials Science and Engineering 21 (2) (2013) 025015.

[42] L.-L. Liu, X.-Z. Wu, R. Wang, W.-G. Li, Q. Liu, Stacking fault energy, yield stress anomaly, and twinnability of Ni 3 Al: A first principles study, Chinese Physics B 24 (7) (2015) 077102.

[43] B. A. Szajewski, A. Hunter, D. J. Luscher, I. J. Beyerlein, The influence of anisotropy on the core structure of Shockley partial dislocations within FCC materials, Modelling and Simulation in Materials Science and Engineering 26 (1) (2018) 015010.

[44] T. Smith, B. Esser, N. Antolin, G. Viswanathan, T. Hanlon, A. Wessman, D. Mourer, W. Windl, D. McComb, M. Mills, Segregation and $\eta$ phase formation along stacking faults during creep at intermediate temperatures in a Ni-based superalloy, Acta Materialia 100 (2015) 19-31.

[45] V. A. Vorontsov, R. E. Voskoboinikov, C. M. Rae, Shearing of $\gamma$ ' precipitates in Ni-base superalloys: A phase field study incorporating the effective $\gamma$-surface, Philosophical Magazine 92 (5) (2012) 608634.

[46] B. Décamps, S. Raujol, A. Coujou, F. Pettinari-Sturmel, N. Clément, D. Locq, P. Caron, On the shearing mechanism of $\gamma^{\prime}$ precipitates by a single $(\mathrm{a} / 6)<112>$ Shockley partial in Ni-based superalloys, Philosophical Magazine 84 (1) (2004) 91-107.

[47] S. Y. Yuan, Z. H. Jiang, J. Z. Liu, Y. Tang, Y. Zhang, Nano-twinning in a $\gamma^{\prime}$ precipitate strengthened Ni-based superalloy, Materials Research Letters 6 (12) (2018) 683-688.

[48] M. Chandran, S. K. Sondhi, First-principle calculation of stacking fault energies in Ni and Ni-Co alloy, Journal of Applied Physics 109 (10) (2011) 103525.

[49] S. Kibey, J. Liu, D. Johnson, H. Sehitoglu, Predicting twinning stress in fcc metals: Linking twinenergy pathways to twin nucleation, Acta Materialia 55 (20) (2007) 6843-6851.

[50] J. Wang, H. Sehitoglu, Dislocation slip and twinning in Ni-based L12 type alloys Intermetallics 52 
(2014) 20-31.

[51] B. Reppich, Some new aspects concerning particle hardening mechanisms in $\gamma^{\prime}$ precipitating Ni-base alloys-I. Theoretical concept Acta Metallurgica 30 (1) (1982) 87-94.

[52] R. E. Voskoboinikov, C. M. F. Rae, A new $\gamma$-surface in $\{111\}$ plane in L12 Ni3 Al IOP Conference Series: Materials Science and Engineering 3 (2009) 012009.

[53] J. Stinville, W. Lenthe, J. Miao, T. Pollock, A combined grain scale elastic-plastic criterion for identification of fatigue crack initiation sites in a twin containing polycrystalline nickel-base superalloy Acta Materialia 103 (2016) 461-473.

[54] A. J. Ardell, J. C. Huang, Antiphase boundary energies and the transition from shearing to looping in alloys strengthened by ordered precipitates, Philosophical Magazine Letters 58 (May) (1988) 189-197.

[55] E. Galindo-Nava, L. Connor, C. Rae, On the prediction of the yield stress of unimodal and multimodal $\gamma^{\prime}$ Nickel-base superalloys Acta Materialia 98 (2015) 377-390.

[56] A. S. Argon, Strengthening Mechanisms in Crystal Plasticity, 1st Edition, Oxford University Press, Oxford, 2008.

[57] R. Kozar, A. Suzuki, W. Milligan, J. Schirra, M. Savage, T. Pollock, Strengthening Mechanisms in Polycrystalline Multimodal Nickel-Base Superalloys, Metallurgical and Materials Transactions A 40 (7) (2009) 1588-1603.

[58] K. Hanson, J. W. Morris, Limiting configuration in dislocation glide through a random array of point obstacles, Journal of Applied Physics 46 (3) (1975) 983-990.

[59] R. B. Schwarz, R. Labusch, Dynamic simulation of solution hardening Journal of Applied Physics 49 (10) (1978) 5174-5187. 